\title{
On Globalization and the Concentration of Talent
}

\author{
Ulrich Schetter and Oriol Tejada \\ CID Research Fellow and Graduate Student \\ Working Paper No. 121 \\ December 2019, revised October 2020
}

(C) Copyright 2019 Schetter, Ulrich; Tejada, Oriol; and the President and Fellows of Harvard College

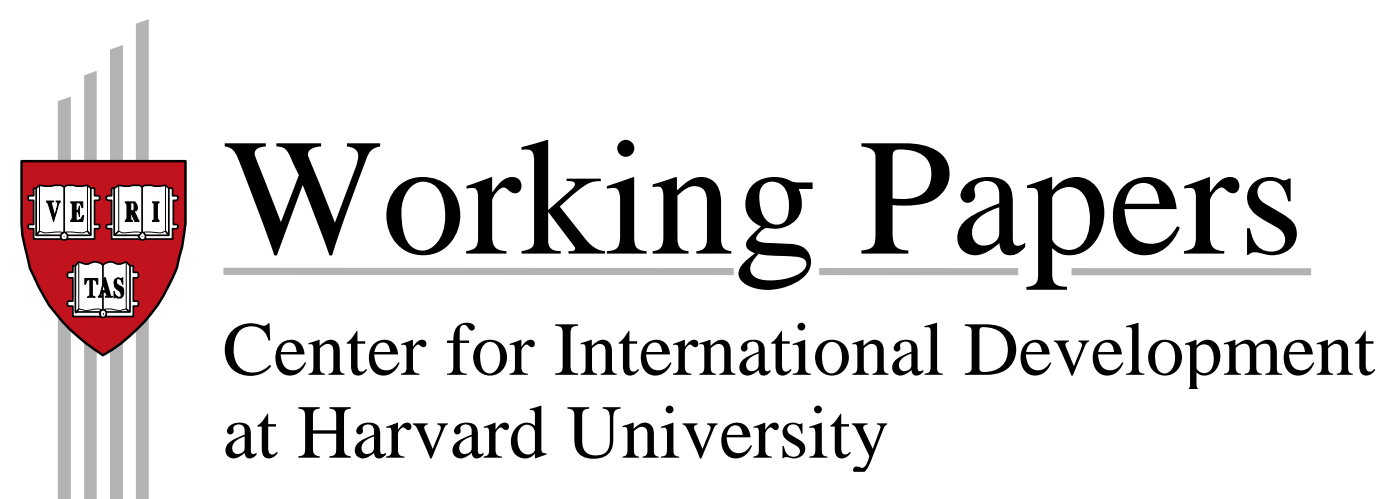




\title{
On Globalization and the Concentration of Talent: A General Result on Superstar Effects and Matching*
}

\author{
Ulrich Schetter \\ Center for International Development \\ at Harvard University \\ Cambridge, MA 02138 \\ ulrich_schetter@hks.harvard.edu
}

\author{
Oriol Tejada \\ CER-ETH \\ Center of Economic Research \\ at ETH Zurich \\ 8092 Zurich, Switzerland \\ toriol@ethz.ch
}

This Version: October 2020

\begin{abstract}
We analyze how globalization affects the allocation of talent across competing teams in large matching markets. Focusing on amplified superstar effects, we show that a convex transformation of payoffs promotes positive assortative matching. This result holds under minimal assumptions on how skills translate into competition outcomes and how competition outcomes translate into payoffs. Our analysis covers many interesting special cases, including simple extensions of Rosen (1981) and Melitz (2003) with competing teams. It also provides new insights on the distributional consequences of globalization, and on the role of technological change, urban agglomeration, and taxation for the composition of teams.
\end{abstract}

Keywords: competing teams, globalization, inequality, matching, superstar effects, technological change, urban agglomeration

JEL Classification: C78, D3, D4, F16, F61, F66, O33

\footnotetext{
${ }^{*}$ We would like to thank Johannes Binswanger, Stefan Bühler, Reto Föllmi, Hans Gersbach, Ricardo Hausmann, Elhanan Helpman, David Hémous, Roland Hodler, Stefan Legge, Georg Nöldeke, Frank Pisch, Josef Zweimüller and seminar participants at the Annual Meeting of the VfS (2018), U St. Gallen, ETH Zurich, U Zurich, the Harvard Growth Lab, and MIT Sloan for helpful comments. Ulrich Schetter gratefully acknowledges financial support from the basic research fund of the University of St. Gallen under grant 1031513.
} 


\section{Introduction}

In recent decades, many countries have seen an increasing segregation of the labor force: high- (low-) skilled workers co-work with other high- (low-) skilled workers more often today than they did in the past (Card et al., 2013; Hakanson et al., 2015; Song et al., 2019). This segregation of the labor force might have profound consequences for income inequality, equal opportunity, political stability, and social cohesion, but still, its causes and consequences are not fully understood. In this paper, we seek to contribute to such understanding by looking at how globalization - and amplified superstar effects more broadly-impacts the concentration of talent among competing teams.

Globalization provides firms with access to new markets. These opportunities, however, do not benefit all firms alike. The most productive firms are the ones that export (Melitz, 2003; Bernard et al., 2007). Similarly, only the best artists or sport teams are able to reach out to global audiences. Teams (or firms) will reach out to foreign markets only if this is profitable. The fact that the best firms serve global markets therefore implies that with globalization total payoffs are more concentrated in the hands of market leaders. We argue that, as a consequence, globalization promotes the concentration of talent. That is, high(low-) skilled workers partner up with other high- (low-) skilled workers more often with globalization than without.

\section{Model}

We build on Chade and Eeckhout (2020) and consider large matching markets where riskneutral workers with heterogeneous skills form competing teams. We then analyze how matching outcomes are affected by globalization. In the baseline model, there are two skill levels (high and low), teams are composed of two workers, and competition among teams results in a rank distribution for each team. The rank distribution is a function of the team's own skill level (i.e., of the skill levels of its members) and of the skill levels of all other teams, which introduces an externality at the matching stage. A team's rank determines its payoff. Later, we show that our main results extend to the case with more skill types and/or more team members and to setups with skill-dependent payoffs instead of rank-dependent payoffs. In such an economy there may be multiple equilibria, some of which may exhibit the property that some, but not all, teams are positively assortatively matched, and we consider the full set of equilibria in our analysis. To fix ideas, however, let us momentarily focus on the case of positive assortative matching (PAM), in which all teams are made up of agents with the same skill level. As is standard in matching models, there is an equilibrium with PAM if the teams' expected payoffs satisfy a supermodularity condition: the expected return of a mixed team - i.e., a team consisting of one high-skilled worker and one low-skilled worker- 
cannot be larger than the average expected return of the teams consisting of only one type of workers. In our model, however, this condition depends on non-trivial interactions between (i) how individual skills translate into team skills, (ii) how a team's own skill level and the skill levels of all other teams in the economy translate into ranks in the competition stage, and (iii) how ranks translate into payoffs. Loosely speaking, it is beneficial to pool talent if either competition outcomes are themselves supermodular - which implies that, on average, a low-skilled team and a high-skilled team reach a higher rank than a mixed team-or if rewards for being ranked atop are very high (superstar effect). In the latter case, an even marginally higher probability for positively assorted teams to reach extreme ranks can suffice to promote PAM regardless of the degree of complementarity between skills.

\section{Main results}

Our main contribution is to show that globalization increases the concentration of talent. We focus on the role of globalization as an amplifier of 'superstar effects' (Rosen, 1981) and define it as a convex transformation of payoffs. ${ }^{1}$ We then prove that under minimal restrictions on the payoff structure and the effect of skills on competition outcomes, the equilibrium with the highest concentration of talent always occurs after globalization, while the equilibrium with the lowest concentration of talent always occurs before globalization. ${ }^{2}$ This implies, in particular, that whenever positive assortative matching is an equilibrium before globalization, it must also be an equilibrium after globalization. The opposite is true for equilibria with negative assortative matching. To show our result, we build on an insight that is long known in the literature on decision-making under uncertainty (Hammond, 1974; Diamond et al., 1974; Jewitt, 1989) and in statistics (Shaked and Shanthikumar, 2007) — see also Karlin et al. (1963). The basic intuition is simple: teams whose members have the same skill level are on average more likely to reach the highest ranks, the ones that benefit most from globalization.

The increase in the concentration of talent has important distributional consequences, and a globalization-induced change in the equilibrium matching has an additional effect on relative wages when compared to the case where workers are always matched positively assorted. The latter case is typically considered in the literature. In fact, we show that under reasonable assumptions on how the rank distribution of a low-skilled team changes with the matching in the overall economy, a globalization-induced increase in the concentration of talent adds to income inequality over and above any potential effect conditional on the matching.

Our main result — globalization leads to more teams being positively assortatively matched-

\footnotetext{
${ }^{1}$ See the literature review in Section 2, as well as our discussions above and in Section 8 for motivations of this view on globalization.

${ }^{2}$ Globalization evolved over time. We therefore use the terms 'before/after globalization' and 'without/with globalization' interchangeably.
} 
is remarkably general. With regard to the competition mode among teams, we simply assume that mixed teams are more likely to be ranked in the mid range, with teams formed only by high-skilled (low-skilled) individuals more likely to be ranked in the upper range (lower range). This puts minimal restrictions on the mode of competition and renders the relationship between skills and performance meaningful. As to the payoff scheme, we only apply the normalization that higher ranks are better and assume accordingly that the payoff scheme is increasing.

Our reduced-form analysis covers many interesting special cases. On the one hand, we show that mixed teams are naturally more likely to achieve mid-range ranks in head-tohead competitions or patent races, for example, as well as in situations with skill-dependent productivity draws from a Pareto distribution. On the other hand, globalization acts as an amplifier of superstar effects in the Rosen (1981) model and the Melitz (2003) model with fixed cost of market entry, for example. This means that our results directly apply to simple extensions of these models encompassing competing teams. More generally, our results apply to environments with amplified superstar effects, which have previously been argued in the literature to arise from various sources other than globalization, including skill-biased technological change, urban agglomeration, and inter-occupational spilloverssee Section 8.3 for a discussion.

\section{Illustrative example: The case of European football}

The causal link we unravel from globalization - and amplified superstar effects in generalto the concentration of talent among competing teams can be illustrated using data from European football leagues. This is a good example for the following reasons: First, football teams compete in their national leagues for rank. Second, a team's performance in these leagues is a direct measure of its skill level relative to the skill levels of the competing teams. Third, each year the clubs ranked highest in their respective national league qualify to participate in the UEFA Champions League (UCL), which provides participating teams with a global platform and large direct payouts from UEFA. Fourth, the enormous growth experienced by the UCL is an instance of our concept of globalization, since as a result of such growth the returns to being ranked high in a national league have dramatically increased over time. ${ }^{3}$

How did this increasing importance of UCL feed back into competition in national leagues?

\footnotetext{
${ }^{3}$ Annual payouts to participating teams, for example, increased more than twentyfold since 1996/97, reaching more than EUR 2bn in 2018/19. Moreover, the final of the UCL is broadcasted in over 200 countries these days, with up to $400 \mathrm{~m}$ people tuning in, making it the biggest annual sports-event worldwide. See https://www.footballbenchmark.com/uefa_champions_league_non_big_five_participation, https://www.uefa.com/uefachampionsleague/news/0250-0c510b7eb8f9-fbe1a8bb6fc2-1000--worldwidereach-of-the-lisbon-final/?referrer $=\% 2$ Fuefachampionsleague $\% 2$ Fnews $\% 2$ Fnewsid $\% 3 D 2111684$, https: //www.uefa.com/uefachampionsleague/news/newsid=2562033.html (retrieved on 14 September 2020).
} 
Figure 1: Concentration of talent in European football leagues

(a) 1st division

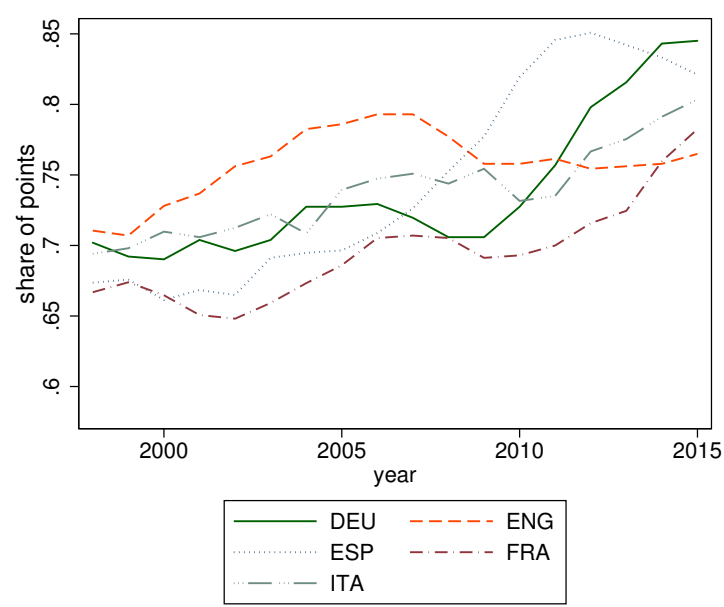

(b) 2nd division

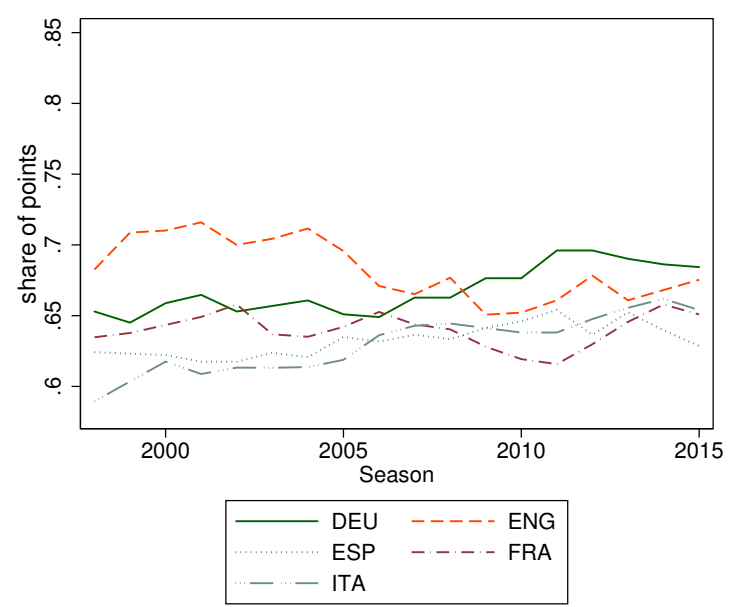

Notes: Own illustration, based on kicker.de and wikipedia.org. The share of points refers to the centered 5 -year moving average of the ratio of end-of-season points of the national champion over the maximum achievable number of points. Panel (a) refers to the respective first-division league for each of the five countries, panel (b) to the corresponding second-division league.

As may be seen from Figure 1(a), this development went hand in hand with an increased concentration of talent in national leagues. This figure shows the share of the maximal achievable points won by the respective national champion for each of the 'big 5' European football leagues - England, France, Germany, Italy, and Spain. This share has been steadily increasing over time, suggesting that the players with the highest talent are increasingly concentrated in a few teams (viz., those who win).

To further substantiate our conjecture that this increase in the concentration of talent is attributable to globalization, we can contrast our 'treatment group' of first-division leagues with the 'control group' of corresponding second-division leagues - see Figure 1(b). ${ }^{4}$ Teams in these leagues cannot qualify for the UCL (or any other European competition) via their national leagues, so globalization is less important for competition in these leagues. If globalization was a key driver for the concentration of talent we observe in first-division leagues, we should not observe the same upward trend in the performance of winning teams in second-division leagues. As shown in Figure 1(b), this is indeed the case.

\section{Organization of the paper}

The remainder of the paper is organized as follows. In Section 2 we review the different strands of the literature that are related to our paper. Section 3 provides a simple example that illustrates our main insights. In Section 4 we introduce the baseline version of our

\footnotetext{
${ }^{4}$ We thank Stefan Legge for sharing this data.
} 
model. In Section 5 we analyze equilibria in our economy. In Section 6 we investigate the effect of globalization on equilibrium outcomes. In Section 7 we consider extensions with several types and team members, alternative modes of competition, a generalization of our main assumption, coalitions of workers, and migration. In Section 8 we present microfoundations for our main assumptions and show that our reduced-form analysis covers interesting special cases from a wide range of fields. Section 9 concludes. The proofs of all the results are in the appendices.

\section{Relation to the Literature}

Our paper is related to several strands of literature.

Superstar effects

In our model, globalization increases the gains from being ranked high in a market, i.e. we think of globalization as an amplifier of superstar effects. In his seminal contribution, Rosen (1981) shows how small differences in the talent of entertainers can result in large heterogeneity in their income if revenues are a convex function of talent. He argues that this is particularly true in markets with imperfect substitutability between artists of different quality and when the marginal cost of reaching out to additional customers is low or even zero as, for example, with performances broadcasted on TV. Hence, in Rosen (1981), superstars benefit from being able to reach broader audiences. ${ }^{5}$ As long as consumption is indivisible in the sense that an increase in quantity cannot compensate for a lower quality, similar effects can, however, also arise if suppliers can serve a fixed number of clients only. Then, increased income inequality on the side of the buyers can translate into income inequality for suppliers. Such mechanisms can explain the increased dispersion in house prices (Määttänen and Terviö, 2014), imply that inequality can spill over across occupations (Gottlieb et al., 2019), and they give rise to higher CEO pay in a globalized world with larger firm sales (Gabaix and Landier, 2008; Terviö, 2008; Gersbach and Schmutzler, 2014; Ma and Ruzic, 2020). These papers have in common that there is always positive assortative matching between buyers' income (or firm size) and suppliers' quality. They carry out comparative statics exercises that can be linked to globalization, keeping the matching constant. While we also investigate comparative statics with regard to globalization that strengthens superstar effects, our model and the main focus of our analysis are very different. We do not consider matching between buyers and sellers, but between workers who form competing teams.

\footnotetext{
${ }^{5}$ Haskel et al. (2012) discuss how globalization can amplify superstar effects and argue based on an augmented Heckscher-Ohlin model that superstar effects á la Rosen (1981) may well have contributed to recent trends in US wage inequality.
} 
We then study the conditions under which equilibrium matchings feature more positive assortativity and show that globalization increases the concentration of talent. In turn, this may fuel (top-) income inequality. Our paper thus complements the literature on superstar effects by showing how they add to seggregation of the labor force and by identifying an additional channel through which they can add to income inequality. ${ }^{6}$

\section{Matching markets}

We build on the literature characterizing matching equilibria. In his seminal contribution, Becker (1973) showed that there is positive assortative matching in a marriage market whenever a couple's payoff function is supermodular in the partners' types (characteristics). ${ }^{7}$ In his paper, payoffs depend exclusively on the own matching. By contrast, we assume that each team's (expected) payoff also depends on the skill levels of all other teams. Specifically, we borrow from Chade and Eeckhout (2020) and study large one-sided matching markets where teams first form and then compete against each other. As noted in Chade and Eeckhout (2020), the competition introduces an externality at the matching stage that can lead to multiple and inefficient equilibria. ${ }^{8}$ Our focus is different. We are concerned neither with uniqueness nor with efficiency of equilibrium outcomes, but with deriving robust comparative statics for the set of equilibrium matchings. ${ }^{9}$ While in one of their (parametric) applications, Chade and Eeckhout (2020) also consider changes in the equilibrium matching - in their case driven by changes in the complementarity between skills-, our (non-parametric) analysis is substantially more general, which enables us to apply our insights to many different contexts - see Section 8. In fact, we show that the comparative statics with regards to the matching in Chade and Eeckhout (2020, Proposition 5) is a reflection of the much more general logic we put forward in this paper.

\section{Globalization}

Our work is also related to the literature analyzing the distributional consequences of globalization more generally. A large literature focuses on international trade. In recent work, trade has been shown to have heterogeneous effects across regional labor markets (e.g. Autor et al. 2013; Dauth et al. 2014; Dix-Carneiro and Kovak 2017), across (types of) workers

\footnotetext{
${ }^{6}$ Our work thus also relates to the broader literature analyzing different drivers of (top-)income inequality, see e.g. Piketty et al. (2014); Bénabou and Tirole (2016); Jones and Kim (2018); Geerolf (2017) for recent theoretical contributions. It also relates, though less closely, to empirical work by Neffke (2019), who analyzes wage effects of coworker matching using Swedish data on detailed educational attainments.

${ }^{7}$ See Kremer (1993); Shimer and Smith (2000); Legros and Newman (2002, 2007); Eeckhout and Kircher (2018) for extensions of these ideas and conditions for positive assortative matching in different contexts.

${ }^{8}$ Another strand of this literature studies existence conditions for stable matchings and efficiency of these matchings in two-sided markets with externalities (Sasaki and Toda, 1996; Hafalir, 2008; Mumcu and Saglam, 2010; Pycia and Yenmez, 2017).

${ }^{9}$ In our baseline setup, we consider the case of pure competition for rank. With total payoff in the market being independent of matching, equilibrium outcomes are trivially efficient.
} 
(e.g. Autor et al. 2014; Galle et al. 2017; Lee 2020; Helpman et al. 2017), and across (types of) consumers (e.g. Faber 2014; Fajgelbaum and Khandelwal 2016). The work by Costinot and Vogel (2010) is somewhat closer to our paper. They consider an assignment model of heterogeneous workers to tasks to study the distributional consequences of international trade. In their model, however, there is always positive assortative matching of workers to tasks. Grossman et al. (2016) consider two-sided matching between managers and workers of different skills that sort into various industries and analyze the distributional effects of changes in the trade environment. While in their setup workers and managers always match in a positively assortative fashion within industries, they may or may not sort in the same fashion across industries, i.e., talent may or may not concentrate in one industry. Grossman et al. (2016), however, do not consider how this concentration itself is affected by globalization, which is our main focus.

Concentration of talent

In this vein, our paper is closer to Kremer and Maskin (2006), Grossman and Rossi-Hansberg (2008), Helpman et al. (2010), and Porzio (2017). These papers are nonetheless very different from ours in terms of the economic environment and the main mechanisms of interest. Helpman et al. (2010) consider a Melitz (2003)-model with search frictions and costly screening in the labor market. They show that trade liberalization increases differences in the average ability of the workforce across firms. In their model abilities are match-specific. Porzio (2017) shows how globalization - in his case, the availability of state-of-the-art technologies in developing countries - can give rise to a dual economy where high-skilled individuals concentrate in the sectors that adopt the state-of-the-art technology. Kremer and Maskin (2006) and Grossman and Rossi-Hansberg (2008) present models of outsourcing of tasks with low skill intensity. Depending on which industries offshore (more) and where the freed-up lowskilled labor gets taken up in the domestic economy, this may also increase the concentration of talent. ${ }^{10}$ These channels and the one we establish between more convex payoffs and more positive assortative matching in the labor force complement each other. Moreover, the generality of our analysis allows to apply our insights to a wide range of contexts, and it speaks to sources of an increased concentration of talent over and above globalization, including urban agglomeration, (skill-biased) technological change, inter-occupational spillovers, and taxation. We discuss these and the related literature in Section 8.3.

\footnotetext{
${ }^{10}$ Maskin (2015) uses the results by Kremer and Maskin (2006) to argue that globalization (i.e., the possibility to trade) leads to an increase in positive assortative matching by increasing the variance of skills.
} 


\section{A Simple Example}

To build intuition for our main results, we begin with a simple example.

Consider a population made up of a continuum of measure two of risk-neutral workers. Half of the individuals are high-skilled and the other half are low-skilled. We investigate the following situation. Individuals form teams of two that then compete against each other, as detailed momentarily, and side-payments are possible. Teams are awarded a payoff $h_{\eta}(y)$, with $\eta \in(0, \infty)$, based on their ranking $y \in[0,1]$ in the competition, where for the purpose of this example we assume the following functional form

$$
h_{\eta}(y):=(1+\eta) \cdot y^{\eta}
$$

Hence, function $h_{\eta}(y)$ is increasing and either concave (if $\eta \leq 1$ ) or convex (if $\eta \geq 1$ ). Moreover, the higher $\eta$, the more convex (or the less concave) is function $h_{\eta}(y)$. This means that if $0<\eta_{1}<\eta_{2}$, then $h_{\eta_{2}}(y)$ can be obtained from $h_{\eta_{1}}(y)$ through an increasing, convex transformation. In addition, note that the average (or total) payoff is 1 irrespective of $\eta$ since

$$
\int_{0}^{1} h_{\eta}(y) d y=1 \text {. }
$$

The (comparative statics) question we want to address in this paper is the following: How does an increase in parameter $\eta$-or, in general, a convex transformation of the payoff scheme - affect matching outcomes and, in particular, does it lead to more positive assortative matching? We shall argue that such a convex transformation of the payoff scheme can be attributed to globalization, among other phenomena.

For the purpose of this example, let us assume that teams compete one-on-one against all other teams in the economy and are then ranked according to their share of wins. There are three possible (types of) teams: a team $t^{h}$ made up of two high-skilled workers; a team $t^{m}$ made up of one high-skilled worker and one low-skilled worker; a team $t^{l}$ made up of two low-skilled workers. If a team of type $t^{h}$ competes against a team of type $t^{l}$, the former wins with probability $p>1 / 2$ and the latter wins with probability $1-p<1 / 2$. If two teams of the same type compete against each other, each of them wins with probability $1 / 2$. Finally, if a team of type $t^{m}$ competes against a team of type $t^{h}\left(t^{l}\right)$, it wins with probability $p_{H}$ $\left(p_{L}\right)$.

When is positive assortative matching (PAM) an equilibrium in this economy? This depends on a familiar supermodularity condition which, in our case, refers to teams' expected payoffs 
in the competition. ${ }^{11}$ Specifically, let us denote the expected (or average) payoff conditional on PAM of a team of type $t^{h}$ by $\mathbb{E}_{h}$ and analogously for teams of type $t^{l}$ and $t^{m}$. As we explain further in Section 5, in an equilibrium with PAM every worker receives half the payoff of his/her team. The necessary and sufficient condition for PAM to be an equilibrium is therefore

$$
\frac{1}{2} \cdot \mathbb{E}_{l}+\frac{1}{2} \cdot \mathbb{E}_{h} \geq \mathbb{E}_{m}
$$

Inequality (2) requires that no worker should find it profitable to match with another worker of a different type and give the latter his/her current payoff.

This inequality depends on two different forces. On the one hand, it depends on how individual skills translate into team skills and how team skills translate into success in the competition. In the example considered here, this is reflected in the parameters $p, p_{L}$, and $p_{H}$. If skills are to be of any value in the competition it should be the case that

$$
0<(1-p)<p_{H}<\frac{1}{2}<p_{L}<p<1
$$

and we make this assumption here. Condition (3) implies that a team of type $t^{h}$ expects to win more often than a team of type $t^{m}$, which, in turn, expects to win more often than a team of type $t^{l}$. Importantly, however, it does not require the probability of winning to be supermodular (or submodular) in skills, which is neither necessary nor sufficient for PAM to be an equilibrium in the set-up considered here. ${ }^{12}$ This is because whether or not PAM is an equilibrium depends on the other hand on how competition outcomes are translated into payoffs, i.e. on the payoff scheme $h_{\eta}(y)$. The latter is impacted by globalization, and it turns out that a globalization-induced convex transformation of the payoff scheme may enable PAM to become an equilibrium, but it can never do the opposite. To see this, note that the fact that teams need to jointly earn the total payoffs leads to

$$
\frac{1}{2} \cdot \mathbb{E}_{l}+\frac{1}{2} \cdot \mathbb{E}_{h}=\int_{0}^{1} h_{\eta}(y) d y=1 .
$$

\footnotetext{
${ }^{11}$ A function $f: X \subset \mathbb{R}^{2} \rightarrow \mathbb{R}$, where $X$ is a lattice of $\mathbb{R}^{2}$, is supermodular (submodular) if $f\left(\max \left\{x_{1}, x_{1}^{\prime}\right\}, \max \left\{x_{2}, x_{2}^{\prime}\right\}\right)+f\left(\min \left\{x_{1}, x_{1}^{\prime}\right\}, \min \left\{x_{2}, x_{2}^{\prime}\right\}\right) \geq(\leq) f\left(x_{1}, x_{2}\right)+f\left(x_{1}^{\prime}, x_{2}^{\prime}\right)$ for $\left(x_{1}, x_{2}\right),\left(x_{1}^{\prime}, x_{2}^{\prime}\right) \in$ $X$.

${ }^{12}$ To see this, suppose for concreteness that all workers are matched positively assorted, i.e. half of the teams are of type $t^{h}$ and half of the teams are of type $t^{l}$. In such a case, supermodularity of the expected probability of winning for a team of type $t^{k}(k \in\{l, m, h\})$, denoted by $Q_{k}$, would require that

$$
\underbrace{\frac{1}{2} \cdot p+\frac{1}{2} \cdot \frac{1}{2}}_{:=Q_{h}}+\underbrace{\frac{1}{2} \cdot(1-p)+\frac{1}{2} \cdot \frac{1}{2}}_{:=Q_{l}} \geq 2 \cdot(\underbrace{\frac{1}{2} \cdot p_{H}+\frac{1}{2} \cdot p_{L}}_{:=Q_{m}})
$$

or, equivalently, that

$$
p_{L}+p_{H} \leq 1
$$

which is clearly not implied by Condition (3).
} 
Moreover, Condition (3) and the law of large numbers ensure that when all teams but one are matched according to PAM, a team of type $t^{m}$ will be ranked at position $y=1 / 2$ with probability one (i.e., above all teams of type $t^{l}$ and below all teams of type $t^{h}$ ), which implies

$$
\mathbb{E}_{m}=(1+\eta) \cdot\left(\frac{1}{2}\right)^{\eta}
$$

Hence, Inequality (2) becomes

$$
M(\eta):=1-(1+\eta) \cdot\left(\frac{1}{2}\right)^{\eta} \geq 0,
$$

which clearly depends on the payoff scheme and does not require the probability of winning to be supermodular. Most importantly, it is a matter of simple algebra to verify that there is $\eta^{*}>0$ satisfying $M\left(\eta^{*}\right)=0$ such that for $\eta>0$,

$$
M(\eta) \geq 0 \Leftrightarrow \eta \geq \eta^{*}
$$

In the remainder of the paper we show that the insight that globalization increases the share of teams that are positively assortatively matched is very general: It applies to matching markets with arbitrary increasing payoff functions, when we consider arbitrary convex transformations, and it holds under minimal restrictions on the mode of competition and on the externalities generated at the matching stage.

\section{Baseline Model}

We build on Chade and Eeckhout (2020) and consider a large economy in which workers of different skills form competing teams. In our baseline model, there are two types of workers that form teams of two. Teams compete for rank, and each rank is awarded a payoff according to some payoff scheme. We then apply a convex transformation of this payoff scheme, and analyze whether as a result of such transformation there is more positive assortative matching. In Section 7 we extend the model in several directions and show the robustness of our results. In what follows, we formally describe our model and its main underlying assumptions, which are further justified in Section 8. For concreteness, we introduce our model with reference to globalization, but our set-up can readily be applied to other contexts - see Section 8.3 for a discussion.

\subsection{Economic environment}

The economy is populated by a continuum of measure two of workers, denoted by $\mathcal{W}$. Workers receive linear utility in money and differ in their skills: they are either high-skilled 
or low-skilled. To simplify the exposition, we assume for now that there is an equal share of each type, but this is not essential - see Section 7.1. All workers of the same type are otherwise indistinguishable from each other. We let $\mathcal{W}=\mathcal{W}^{l} \cup \mathcal{W}^{h}$, where $\mathcal{W}^{h}$ and $\mathcal{W}^{l}$ denote the set of high-skilled and low-skilled workers, respectively, which are both of measure 1 . Workers match to other workers and form teams, which consist of a pair of workers and are generally denoted by $t$. A team may be of three types: two high-skilled workers may match, and we use $t^{h}$ to denote such a team; two low-skilled workers may match, and we use $t^{l}$ to denote such a team; one worker of each type may match, and we use $t^{m}$ to denote such a team. Teams differ in their overall skill level, which is weakly increasing in the skills of each team member. Side-payments are possible, and thus we consider an environment with transferable utility.

A matching $\mu$ is the collection of all teams. That is, each worker belongs to exactly one team, and thus a matching partitions the set $\mathcal{W}$. We denote the set of all possible matchings of $\mathcal{W}$ by $\mathcal{M}$. Except for changes that affect sets of workers of measure zero, $\mathcal{M}$ can be indexed in our setup by $\mu(\alpha)$ with $\alpha \in[0,1]$, where $(1-\alpha)$ denotes the fraction of all teams in $\mu(\alpha)$ that are $t^{m}$. In turn, $\alpha / 2$ denotes the fraction of all teams that are $t^{h}$ and $t^{l}$, respectively. We refer to $\mu(1)$ as the positive assortative matching (PAM). Similarly, we refer to $\mu(0)$ as the negative assortative matching (NAM).

\subsection{Competition between teams}

Teams compete for rank, since their payoff depends only on their rank $y \in[0,1]$. We apply the convention that rank $y=1(y=0)$ is the best (the worst). The exact nature of the competition does not matter for the purpose of the paper, and for now it suffices to assume that competition results in some rank distribution for each team. This rank distribution depends on a team's own skill level, as well as on the skill levels of all other teams in the economy. As in Chade and Eeckhout (2020), there is therefore an externality at the matching stage, since changes in the matching feed back into the rank distributions for different types of teams and, hence, into the expected payoffs of these teams. ${ }^{13}$ We use $F^{\alpha, k}(y)$ to denote the probability that given a matching $\mu(\alpha)$, with $\alpha \in[0,1]$, a team $t^{k}$ reaches rank $y$ or lower, with $k \in\{l, m, h\}$. Then we define the competition as a set of rank distributions for each team type $k \in\{l, m, h\}$ and each matching $\mu(\alpha), \mathcal{F}=\left\{\left(F^{\alpha, l}, F^{\alpha, m}, F^{\alpha, h}\right)\right\}_{\alpha \in[0,1]}$.

For simplicity, we assume that for all $\alpha \in[0,1]$ and all $k \in\{l, m, h\}, F^{\alpha, k}(\cdot)$ is continuously differentiable, and at times we denote the corresponding probability distribution function

\footnotetext{
${ }^{13}$ We allow these externalities to play out arbitrarily, only restricting them by the weak conditions laid out in Assumptions 1 and 4 below.
} 
(PDF) by $f^{\alpha, k}(y):=\frac{d F^{\alpha, k}(y)}{d y} \cdot{ }^{14}$ Because all positions in the ranking have to be filled, we must have that for all $y \in[0,1]$

$$
1=\frac{\alpha}{2} \cdot f^{\alpha, l}(y)+\frac{\alpha}{2} \cdot f^{\alpha, h}(y)+(1-\alpha) \cdot f^{\alpha, m}(y) .
$$

To render the notion of skill meaningful, we define for all $\alpha \in[0,1]$

$$
\mathcal{A}_{\alpha}^{\mathcal{F}}:=\left\{y \in[0,1]: f^{\alpha, m}(y) \geq 1\right\}
$$

and

$$
\tilde{\mathcal{A}}_{\alpha}^{\mathcal{F}}:=\left\{y \in[0,1]: f^{\alpha, l}(y)+f^{\alpha, h}(y) \leq 2\right\} .
$$

Then consider the following condition:

\section{Assumption 1 (Competition)}

Sets $\mathcal{A}_{\alpha}^{\mathcal{F}}$ and $\tilde{\mathcal{A}}_{\alpha}^{\mathcal{F}}$ are convex and compact for all $\alpha \in[0,1]$.

Due to Equation (5), we have $\mathcal{A}_{\alpha}^{\mathcal{F}}=\tilde{\mathcal{A}}_{\alpha}^{\mathcal{F}}$ for all $\alpha \in(0,1)$. Further, if rank distributions are continuous in the distributions of skills in the entire economy, the boundaries of the sets $\mathcal{A}_{\alpha}^{\mathcal{F}}$ and $\tilde{\mathcal{A}}_{\alpha}^{\mathcal{F}}$ are continuous in $\alpha .{ }^{15}$ Any restrictions on the sets $\mathcal{A}_{\alpha}^{\mathcal{F}}$ and $\tilde{\mathcal{A}}_{\alpha}^{\mathcal{F}}$ are then a reflection of the same underlying rationale, and we introduce this distinction to avoid technical difficulties pertaining to the limiting behavior of the various rank distributions when analyzing equilibria with positive and negative assortative matching, respectively.

For further understanding of the economic content of Assumption 1, consider the case of some $\alpha \in(0,1)$. Since $f^{\alpha, m}(y)$ is continuous in $[0,1]$, the set $\mathcal{A}_{\alpha}^{\mathcal{F}}$ is compact. This is a standard assumption of a technical nature. The more interesting part of Assumption 1 is that the set $\mathcal{A}_{\alpha}^{\mathcal{F}}$ is convex. This is a minimal definition of skills in our economy. It is satisfied if $f^{\alpha, m}(\cdot)$ is monotonic or quasi-concave, and in particular if it is single-peaked. More generally, it is satisfied if a mixed team is likely to achieve ranks in the mid range. To see this, consider that matching $\mu(\alpha)$ has formed, i.e., there are shares $1-\alpha$ of mixed teams and $\alpha / 2$ of low- and high-skilled teams, respectively. What should each of the mixed teams expect in terms of its ranking in the competition? One possibility is that $f^{\alpha, m}(y)=1$ for all $y \in[0,1]$, in which case $\mathcal{A}_{\alpha}^{\mathcal{F}}=[0,1]$ and all such teams expect to be ranked in any position with the same probability. In general, $\mathcal{A}_{\alpha}^{\mathcal{F}}$ is the set of ranks whose associated probability for a mixed team is at least that of the former uniform case. This determines in itself a plausible threshold that separates ranks $y \in[0,1]$ into low-probability ranks $\left(f^{\alpha, m}(y)<1\right)$ and high-probability ranks $\left(f^{\alpha, m}(y) \geq 1\right)$.

By assuming that $\mathcal{A}_{\alpha}^{\mathcal{F}}$ is a convex set, we rule out competitions whose outcome does not behave naturally with regard to the rank of the mixed teams. Indeed, if skills of the members

\footnotetext{
${ }^{14}$ This assumption can easily be dispensed with - see Section 7.3.

${ }^{15}$ Our subsequent analysis does not hinge on this assumption.
} 
Figure 2: Illustration of Assumption 1 for $\alpha=1$

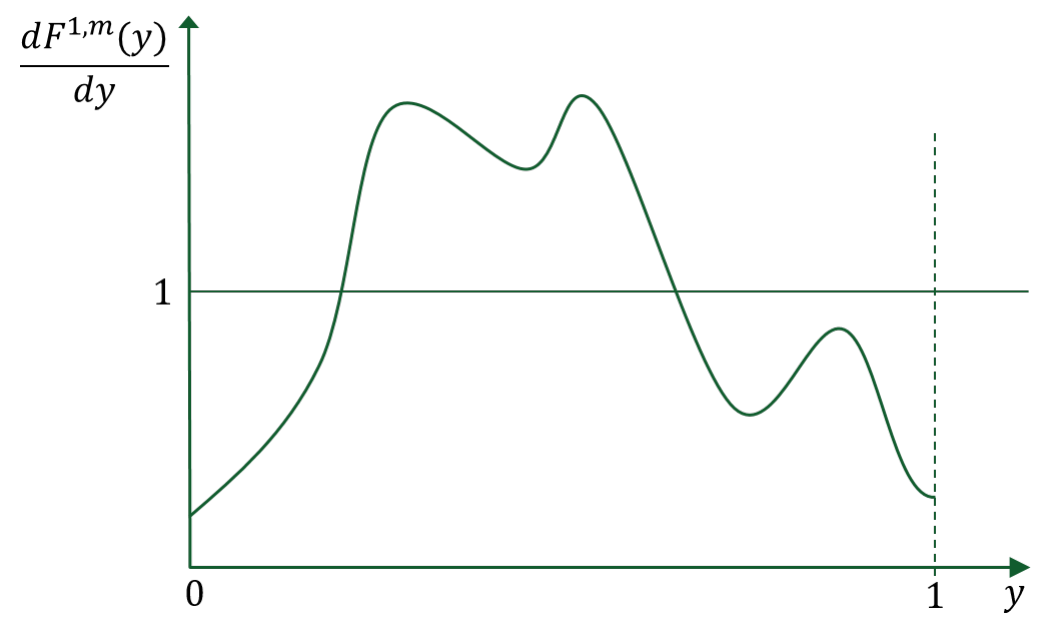

of a team are to yield an advantage for the ranking in the competition, mixed teams should expect to be ranked in mid-range positions more often than in the uniform case, with lowskill teams being ranked low and high-skill teams being ranked high more often than in the uniform case. A weak way of implementing this rationale is to preclude the possibility that for a mixed team a low-probability rank exists between two high-probability ranks. This is captured in Assumption 1 and illustrated by Figure 2 for the case of $\alpha=1$.

Finally, we note that Assumption 1 is naturally satisfied in many economic applications, including head-to-head competitions, patent races, and situations with random productivity draws. We document this in Section 8.1.

\subsection{Payoffs}

Teams receive rank-dependent payoffs. We let

$$
\begin{aligned}
h:[0,1] & \rightarrow \mathbb{R}_{+} \\
y & \rightarrow h(y)
\end{aligned}
$$

denote the payoff scheme that assigns a certain amount of money $h(y)$ to the team that has been ranked in position $y \in[0,1]$. Remaining consistent with our normalization that higher ranks are better, we assume that payoffs are weakly increasing in rank.

\section{Assumption 2 (Payoffs)}

(i) $h(0)=0$,

(ii) $h^{\prime}(\cdot) \geq 0$.

The normalization $h(0)=0$ can be made without loss of generality. We let

$$
\mathcal{H}=\left\{h:[0,1] \rightarrow \mathbb{R}_{+}: h \in C^{1}([0,1]), h(0)=0, h^{\prime}(\cdot) \geq 0\right\}
$$


denote the set of admissible payoff schemes, where $C^{1}([0,1])$ denotes the set of continuously differentiable functions on $[0,1]$.

\subsection{Globalization}

Our main focus is on the redistributive aspects of globalization and on how these interact with the concentration of talent. To that end, we consider a reduced-form modeling choice for globalization, which is micro-founded in Section 8.2. Specifically, we represent (the effect of) globalization by a twice continuously differentiable function

$$
\begin{aligned}
g: \mathbb{R}_{+} & \rightarrow \mathbb{R}_{+} \\
x & \rightarrow g(x),
\end{aligned}
$$

which modifies the payoff scheme and transforms it from $h$ to $g \circ h .{ }^{16}$ We assume that $g(\cdot)$ satisfies the following restrictions:

\section{Assumption 3 (Globalization)}

(i) $g(0)=0, \quad$ (ii) $g^{\prime}(0) \geq 0, \quad$ (iii) $g^{\prime \prime}(\cdot) \geq 0$.

Assumptions 3(i) and 3(ii) are mainly technical in nature. They simply guarantee that $g \circ h \in \mathcal{H}$ for all $h \in \mathcal{H}$ and all $g \in \mathcal{G}$, where $\mathcal{G}$ is the set of functions satisfying Assumption 3. Assumption 3(iii) is more substantive. It implies that globalization takes the form of a convex transformation of the payoff scheme, which is also increasing thanks to Assumption 3(ii). The latter feature preserves the normalization that higher ranks are better, while the increased convexity of payoffs reflects an amplified 'superstar effect', as already noted by Rosen (1981). Assumption 3 is further justified in Section 8.2 - see also Section 8.3.

\section{$5 \quad$ Equilibrium}

In the previous section we outlined the baseline version of our model. To sum up, we are considering an economy that is characterized by a triple $e=(\mathcal{W}, h, \mathcal{F})$, composed of a set of workers $\mathcal{W}$, a payoff scheme $h(\cdot)$, and a competition $\mathcal{F}$. We now proceed with the analysis of equilibria in each economy $e \in \mathcal{E}$, where we use $\mathcal{E}$ to denote the set of all possible economies that satisfy our previous restrictions. We start by characterizing the potential equilibria and then discuss their general existence. The proofs of the results of this section are based on Chade and Eeckhout (2020) and can be found in Appendix B.

\footnotetext{
${ }^{16}$ Our approach to modelling globalization is similar in spirit to the approach taken e.g. by Grossman et al. (2016), who model globalization - a change in the trade environment in their case - as a change in relative output prices.
} 


\subsection{Characterization of equilibria}

Given $e=(\mathcal{W}, h, \mathcal{F}) \in \mathcal{E}$, it is useful to define, for $k \in\{l, m, h\}$,

$$
V\left(t^{k} \mid \mu(\alpha)\right):=\int_{0}^{1} h(y) d F^{\alpha, k}(y)
$$

That is, $V\left(t^{l} \mid \mu(\alpha)\right), V\left(t^{m} \mid \mu(\alpha)\right)$, and $V\left(t^{h} \mid \mu(\alpha)\right)$ denote the expected payoff of a team $t^{l}, t^{m}$, and $t^{h}$, respectively, in economy $e$ when teams are arranged according to $\mu(\alpha) .{ }^{17}$ Now, remember that we are assuming linear utility in money, so workers care about their expected payoffs only. Further, with a continuum of workers, we can see them as operating in a competitive labor market. This means that workers can decide to form teams with other workers in the economy, taking as given the wage rates for skilled and unskilled workers, which we denote by $\bar{w}$ and $\underline{w}$, respectively. ${ }^{18}$ An equilibrium in an economy $e$ is therefore defined as follows:

\section{Definition 1 (Equilibrium)}

An equilibrium of economy $e \in \mathcal{E}$ is a triple $(\mu(\alpha), \underline{w}, \bar{w}) \in \mathcal{M} \times \mathbb{R} \times \mathbb{R}$ such that, for $k \in\{l, m, h\}$,

$$
t^{k} \in \mu(\alpha) \Rightarrow \begin{cases}V\left(t^{h} \mid \mu(\alpha)\right)-\bar{w} \geq V\left(t^{m} \mid \mu(\alpha)\right)-\underline{w} & \text { if } k=h \\ V\left(t^{m} \mid \mu(\alpha)\right)-\bar{w} \geq V\left(t^{l} \mid \mu(\alpha)\right)-\underline{w} & \text { and } \\ V\left(t^{m} \mid \mu(\alpha)\right)-\underline{w} \geq V\left(t^{h} \mid \mu(\alpha)\right)-\bar{w} & \text { if } k=m \\ V\left(t^{l} \mid \mu(\alpha)\right)-\underline{w} \geq V\left(t^{m} \mid \mu(\alpha)\right)-\bar{w} & \text { if } k=l .\end{cases}
$$

That is, within any team matched under $\mu(\alpha)$, none of its members expects a higher payoff by forming another team with a worker of a type different from his/her current match. In other words, in an equilibrium there are no incentives for workers to break away from the current pair and form a new pair. This captures the standard notion of pairwise stability in the matching literature (see e.g. Gale and Shapley, 1962; Chung, 2000). When $\alpha=1$, the several conditions encapsulated in (10) reduce to

$$
\begin{aligned}
& V\left(t^{h} \mid \mu(1)\right)-\bar{w} \geq V\left(t^{m} \mid \mu(1)\right)-\underline{w} \text { and } \\
& V\left(t^{l} \mid \mu(1)\right)-\underline{w} \geq V\left(t^{m} \mid \mu(1)\right)-\bar{w} .
\end{aligned}
$$

The next result characterizes equilibria in our economy.

\footnotetext{
${ }^{17}$ To simplify notation, we drop the dependence of $V(\cdot \cdot \cdot)$ on $e$.

${ }^{18}$ In our economy, teams' payoffs, and hence, payoffs to team members, are random. Since utility is linear in money, workers are risk-neutral and therefore $\bar{w}(\underline{w})$ is just the payoff that a high-skilled (low-skilled) worker expects to receive.
} 


\section{Proposition 1 (Equilibrium)}

Let $e=(\mathcal{W}, h, \mathcal{F}) \in \mathcal{E}$. Then the following statements hold:

(i) There is an equilibrium with positive assortative matching (PAM, $\alpha=1)$ if and only if

$$
\int_{0}^{1} h(y) d y \geq \int_{0}^{1} h(y) d F^{1, m}(y) .
$$

In this case, the equilibrium wages are

$$
\begin{aligned}
& \bar{w}=\frac{1}{2} \cdot \int_{0}^{1} h(y) d F^{1, h}(y) \\
& \underline{w}=\frac{1}{2} \cdot \int_{0}^{1} h(y) d F^{1, l}(y) .
\end{aligned}
$$

(ii) There is an equilibrium with $\alpha \in(0,1)$ if and only if

$$
\int_{0}^{1} h(y) d y=\int_{0}^{1} h(y) d F^{\alpha, m}(y) .
$$

In this case, the equilibrium wages are

$$
\begin{aligned}
& \bar{w}=\frac{1}{2} \cdot \int_{0}^{1} h(y) d F^{\alpha, h}(y) \\
& \underline{w}=\frac{1}{2} \cdot \int_{0}^{1} h(y) d F^{\alpha, l}(y) .
\end{aligned}
$$

(iii) There is an equilibrium with negative assortative matching (NAM, $\alpha=0)$ if and only if

$$
\int_{0}^{1} h(y) d y \geq \int_{0}^{1} h(y) \cdot\left(\frac{1}{2} d F^{0, h}(y)+\frac{1}{2} d F^{0, l}(y)\right) .
$$

In this case, the equilibrium wages satisfy

$$
\begin{aligned}
& \bar{w} \in\left[\frac{1}{2} \cdot \int_{0}^{1} h(y) d F^{0, h}(y), \int_{0}^{1} h(y) d F^{0, m}(y)-\frac{1}{2} \cdot \int_{0}^{1} h(y) d F^{0, l}(y)\right] \\
& \underline{w}=\int_{0}^{1} h(y) d F^{0, m}(y)-\bar{w} .
\end{aligned}
$$

\section{Proof:}

See Appendix B.1.

Our subsequent analysis revolves around Conditions (13), (16), and (19) and how these are affected by globalization. For instance, take Condition (13), which is necessary and sufficient for an equilibrium with positive assortative matching to exist. Because in an 
equilibrium with PAM there are only high- and low-skilled teams in equal shares and on average they must earn the expected payoff across all teams in the economy, Condition (13) can be rewritten as

$$
\int_{0}^{1} h(y) \cdot \frac{1}{2} \cdot\left[d F^{1, l}(y)+d F^{1, h}(y)\right] \geq \int_{0}^{1} h(y) \cdot d F^{1, m}(y) .
$$

This is a standard supermodularity condition. The key observation is that, in our model, supermodularity refers to expected payoffs, which are determined by non-trivial interactions between the competition mode - as reflected in $d F^{1, k}$ —and the payoff scheme - as captured by $h(y)$. The latter is affected by globalization. Hence, the condition that a team's expected rank - which itself depends on how individual skills translate into team skills and on how team skills translate into competition outcomes - is supermodular, namely

$$
\int_{0}^{1} y \cdot \frac{1}{2} \cdot\left[d F^{1, l}(y)+d F^{1, h}(y)\right] \geq \int_{0}^{1} y \cdot d F^{1, m}(y),
$$

is neither necessary nor sufficient for positive assortative matching to be an equilibrium when the payoff scheme is $h(y)$.

Finally, note the generality of our setup with respect to the equilibrium conditions of interest, namely, Conditions (13), (16), (19). We apply the normalization that higher ranks are better $\left(h^{\prime}(y) \geq 0\right)$ and we build on our weak notion of skills in the competition as discussed in Section 4.2. Other than that, we do not impose any restrictions on how workers' skills translate into team skills, on how a team's own skill level and the skill levels of all other teams translate into competition outcomes, and on how competition outcomes translate into payoffs.

\subsection{Existence}

After characterizing potential equilibria, we want to investigate which conditions on the expected payoffs guarantee that an equilibrium exists. To this end, we henceforth impose the following condition:

\section{Assumption 4 (Existence of equilibrium)}

$V\left(t^{k} \mid \mu(\alpha)\right)$ is continuous in $\alpha$, for $k \in\{l, m, h\}$.

Assumption 4 demands the (expected) payoff of each team to depend continuously on changes in the workers' matching. A sufficient, but not necessary, condition for this to happen is that the competition outcome itself does not change abruptly when small changes occur in the way workers match in the economy, i.e., that $f^{\alpha, k}$ is continuous in $\alpha$. Under this 
mild assumption, mixed teams' (expected) payoffs also depend continuously on the matching, both without and with globalization. It turns out that an equilibrium always exists in this scenario, in which case it is characterized by Proposition 1.

\section{Proposition 2}

Let $e=(\mathcal{W}, h, \mathcal{F}) \in \mathcal{E}$. Then an equilibrium exists.

\section{Proof:}

See Appendix B.2.

The above proposition ensures that an equilibrium exists but does not guarantee that it is unique. In our set-up, the competition between teams introduces an externality at the matching stage, and this can give rise to multiple equilibria (Chade and Eeckhout, 2020). Uniqueness can nevertheless be guaranteed when imposing further restrictions on expected payoffs - see Appendix C.1. These conditions are not needed for our subsequent analyses, but they may help in interpreting our main result.

\section{Main Results}

In this section we first analyze how globalization impacts the concentration of talent and then discuss how this affects equilibrium wages.

\subsection{Globalization and the concentration of talent}

To analyze how globalization impacts the concentration of talent, we ask how it affects Equilibrium Conditions (13), (16), and (19) in Proposition 1, which are necessary and sufficient for $\alpha=1, \alpha \in(0,1)$, and $\alpha=0$ to be an equilibrium, respectively. Because these conditions are invariant with respect to positive affine transformations of the payoff scheme, we henceforth normalize payoffs without loss of generality so that

$$
\int_{0}^{1} h(y) d y=\int_{0}^{1} g(h(y)) d y .
$$

Equation (22) highlights the importance of the redistributive aspects of globalization for our analysis.

As noted above, there may be multiple equilibria. Accordingly, let $\underline{\alpha}_{h}\left(\bar{\alpha}_{h}\right)$ denote the equilibrium with the lowest (highest) concentration of talent without globalization and 
analogously for $\underline{\alpha}_{g}\left(\bar{\alpha}_{g}\right)$. The latter refers to the case with globalization. With this notation at hand, we are in a position to state our main result regarding the (global) effect of globalization on the concentration of talent.

\section{Theorem 1}

Let $e=(\mathcal{W}, h, \mathcal{F}) \in \mathcal{E}$ and $g \in \mathcal{G}$. Then

(i) $\underline{\alpha}_{h} \leq \underline{\alpha}_{g}$

(ii) $\bar{\alpha}_{h} \leq \bar{\alpha}_{g}$.

Moreover, each of the two inequalities is sometimes strict.

\section{Proof:}

See Appendix A.

The above theorem provides clear-cut predictions regarding the effect of globalization on the concentration of talent. It says that no matter the set of equilibria that exist with and without globalization, it must always be the case that the equilibrium with the highest (lowest) concentration of talent is one with (without) globalization. Hence, as a result of globalization, the concentration of talent can always weakly increase by shifting the economy from an equilibrium with $\mu(\alpha)$ to an equilibrium with $\mu\left(\alpha^{\prime}\right)$, where $\alpha, \alpha^{\prime} \in[0,1]$ satisfy $\alpha \leq \alpha^{\prime}$. Whenever the equilibrium is unique, this is necessarily the case.

To obtain Theorem 1, we have imposed two conditions: First, a team's expected payoff depends continuously on the economy-wide matching, as ensured by Assumption 4. This assumption is mostly technical in nature. Second, a mixed team is more likely to achieve mid-range ranks in economies no matter the share of teams that are matched positively assorted, as ensured by Assumption 1. This is a very mild assumption requiring skills to be meaningful. ${ }^{19}$ The basic intuition is the following: Globalization rewards teams ranked high in the market. When compared to a mixed team, teams whose members are matched positively assorted are more likely to achieve extreme ranks and, in particular, to be ranked at the top. This is because the rank distribution of the mixed teams is biased towards achieving mid-range ranks, while low-skilled teams (high-skilled teams) are biased towards

\footnotetext{
${ }^{19}$ As may be seen from Appendix A, this allows applying a well-known result from the literature on decision-making under uncertainty (Hammond, 1974; Diamond et al., 1974; Jewitt, 1989) and statistics (Karlin et al., 1963; Shaked and Shanthikumar, 2007). To see the connection, note that we can interpret any payoff scheme $h(y)$ - and $g(h(y))$, for that matter - as a Bernoulli utility function, and the rank distributions - as well as the uniform distribution - as lotteries. We are extremely grateful, without implicating, to Georg Nöldeke for pointing out this analogy to us and for very helpful guidance on the related literature.
} 
achieving low-range ranks (high-range ranks). In other words, teams whose members are matched positively assorted will (on average) benefit more from a globalization-induced amplified 'superstar effect'.

From Theorem 1 two corollaries follow easily, which focus on cases that feature prominently in the literature, and which allow us to illustrate our main result in a clear way. For this purpose, we say that an economy $e \in \mathcal{E}$ satisfies PAM (satisfies $N A M$ ) if there are wages $\underline{w}$ and $\bar{w}$ such that $(\mu(1), \underline{w}, \bar{w})((\mu(0), \underline{w}, \bar{w}))$ is an equilibrium of economy $e$. From Proposition 1 we know that this is the case if and only if $h(\cdot)$ and $\mathcal{F}$ satisfy Condition (13) (Condition (19)). Note that PAM $(\alpha=1)$ and NAM $(\alpha=0)$ represent two extremes of the one-dimensional space $\{\mu(\alpha)\}_{\alpha \in[0,1]}$.

\section{Corollary 1}

Let $e=(\mathcal{W}, h, \mathcal{F}) \in \mathcal{E}$ and $g \in \mathcal{G}$. Then,

(i) if $(\mathcal{W}, h, \mathcal{F})$ satisfies PAM, so does $(\mathcal{W}, g \circ h, \mathcal{F})$;

(ii) sometimes $(\mathcal{W}, g \circ h, \mathcal{F})$ satisfies PAM and $(\mathcal{W}, h, \mathcal{F})$ does not.

This first corollary states that whenever there is an equilibrium with positive assortative matching prior to globalization, such an equilibrium also exists with globalization. Moreover, in some cases an equilibrium with PAM exists with globalization but not without. In other words, globalization promotes the emergence of PAM as an equilibrium. It is worth noting that this result relies only on the case of $\alpha=1$ in Assumption 1 and not at all on Assumption 4 .

While Corollary 1 analyzes the existence of equilibria with positive assortative matching, the concentration of talent also increases when moving out of an equilibrium with negative assortative matching. It turns out that globalization also promotes the concentration of talent in this latter sense, as shown by the second corollary.

\section{Corollary 2}

Let $e=(\mathcal{W}, h, \mathcal{F}) \in \mathcal{E}$ and $g \in \mathcal{G}$. Then,

(i) if $(\mathcal{W}, g \circ h, \mathcal{F})$ satisfies $N A M$, so does $(\mathcal{W}, h, \mathcal{F})$;

(ii) sometimes $(\mathcal{W}, h, \mathcal{F})$ satisfies $N A M$ and $(\mathcal{W}, g \circ h, \mathcal{F})$ does not.

To sum up, Theorem 1 together with Corollaries 1 and 2 reveal that our theory predicts strong implications of globalization for the concentration of talent. This has important distributional consequences, as we discuss next. 


\subsection{Globalization and wage inequality}

Although our main interest is to understand how globalization impacts matching outcomes, it is worth mentioning that a globalization-induced increased concentration of talent has distributional consequences. It is well known that conditional on positive assortative matching, globalization can increase (decrease) relative wages of high-skilled (low-skilled) workers via amplified superstar effects. ${ }^{20}$ While under plausible restrictions this is also the case here, our setup is general enough to accommodate situations where this direct effect of globalization on wages might not take place. ${ }^{21}$ More importantly, in our setup, there is an additional, indirect effect that occurs because the matching might also change as a result of globalization. To see this, note that the overall wage effect for low-skilled workers can be decomposed as follows

$$
\begin{aligned}
& \frac{1}{2} \cdot \int_{0}^{1} g(h(y)) d F^{\alpha_{g}, l}(y)-\frac{1}{2} \cdot \int_{0}^{1} h(y) d F^{\alpha_{h}, l}(y) \\
= & {\left[\frac{1}{2} \cdot \int_{0}^{1} g(h(y)) d F^{\alpha_{g}, l}(y)-\frac{1}{2} \cdot \int_{0}^{1} h(y) d F^{\alpha_{g}, l}(y)\right] } \\
+ & {\left[\frac{1}{2} \cdot \int_{0}^{1} h(y) d F^{\alpha_{g}, l}(y)-\frac{1}{2} \cdot \int_{0}^{1} h(y) d F^{\alpha_{h}, l}(y)\right], }
\end{aligned}
$$

and analogously for the wage of the high-skilled workers. In the above equation, we have used $\alpha_{g}\left(\alpha_{h}\right)$ to denote the matching outcome with (without) globalization, and we have assumed that $\alpha_{g} \geq \alpha_{h}>0$, for simplicity, so that there is no NAM. The first term on the right-hand side of Equation (23) captures the effect of globalization conditional on the matching. The second term in brackets captures the additional effect that arises from a potential change in the matching, i.e. it is the change in the wage of the low-skilled workers conditional on the payoff scheme.

Now recall that for $\alpha>0$ the equilibrium conditions of Proposition 1 refer to the payoff distribution of mixed teams, but do not directly refer to the payoff distributions of highskilled teams or low-skilled teams. The latter distributions nonetheless determine the wages. This means that the sign of both terms on the right-hand side of Equation (23) is ambiguous, unless more structure is imposed. Yet, plausible restrictions allow for stronger comparative statics. An interesting case is one where $F^{\alpha_{h}, l}(y) \leq F^{\alpha_{g}, l}(y)$ for all $y \in[0,1]$, i.e. if $F^{\alpha_{h}, l}(y)$ first-order stochastically dominates (FOSD) distribution $F^{\alpha_{g}, l}(y)$. In this case, the second term on the right-hand side of Equation (23) is negative. ${ }^{22}$ This suggests that in a world

\footnotetext{
${ }^{20} \mathrm{Cf}$. the literature review in Section 2 .

${ }^{21}$ This is in line with Chade and Eeckhout (2020) analysis on wage inequality (dispersion), as only under some parametric restrictions they can find comparative statics results.

${ }^{22} \mathrm{FOSD}$ is necessary for the second term on the right-hand side of Equation (23) to be negative for every increasing $h(y)$ (Mas-Colell et al., 1995, Proposition 6.D.1).
} 
where low-skilled teams are relatively better at competing against mixed teams than against assortatively matched teams, globalization has a negative effect on the relative wages of the low-skilled workers over and above any potential effect conditional on the matching. ${ }^{23}$ We summarize these insights in the following proposition. ${ }^{24}$

\section{Proposition 3}

Let $\alpha_{g}\left(\alpha_{h}\right)$ denote the equilibrium matching with (without) globalization and suppose that $F^{\alpha_{h}, l}(y) \leq F^{\alpha_{g}, l}(y)$ for all $y \in[0,1]$. Then globalization adds to income inequality over and above any effect conditional on the matching.

\section{Beyond the Baseline Model}

In this section we discuss several generalizations and variations of our baseline setup and show that our main results readily apply to these alternative settings. First, we consider several types and team members. Second, we consider markets where teams do not compete for rank. Third, we consider core allocations. Fourth, we consider a generalization of Assumption 1. Fifth and last, we discuss migration. For simplicity, we focus on Corollary 1, but most of our insights carry over to Theorem 1.

\subsection{Several types and team members}

We start by analyzing how Corollary 1 can be extended to the case with several skill types and several team members. ${ }^{25}$ Suppose there are $S \geq 2$ types with arbitrary population shares, which can be identified by their skill level $s \in \mathcal{S}$, where $\mathcal{S}$ denotes the set of skills available in the economy. Let $N \geq 2$ denote the number of workers in each team $t$, with $\mathcal{S}_{t}=$ $\left\{s_{t}^{1}, s_{t}^{2}, \ldots s_{t}^{N}\right\}$ denoting the multiset of skill levels in team $t$. Then, there is an equilibrium with positive assortative matching if and only if for every worker type $s \in \mathcal{S}$,

$$
V\left(t^{\{s\}^{N}} \mid P A M\right)-(N-1) \cdot w^{s} \geq \max _{\hat{\mathcal{S}} \in \mathcal{S}^{N-1}}\left\{V\left(t^{s \cup \hat{\mathcal{S}}} \mid P A M\right)-\sum_{\hat{s} \in \hat{\mathcal{S}}} w^{\hat{s}}\right\} .
$$

\footnotetext{
${ }^{23} \mathrm{An}$ increased concentration of talent may well have distributional consequences over and above any immediate wage effect, e.g. in the presence of knowledge spillovers. A thorough investigation of such effects is beyond the scope of our paper and is left for future research.

${ }^{24}$ In the previous discussions, we ruled out that $\alpha_{h}=0$. Nevertheless, the fact that in an equilibrium with NAM $\underline{w}$ is at least half the expected payoff of a low-skilled team given NAM (see Equations (20) and (21)) directly implies that the result extends to any economy where $\alpha_{g}>\alpha_{h}=0$.

${ }^{25}$ With multiple types and team members, the notion of mixed equilibria becomes unclear. Hence, focusing on Corollary 1 is not only for simplicity here, but it also covers the case where our results are unambiguous.
} 
Here, $w^{s}$ denotes the equilibrium wage for a worker with skill level $s, t^{\mathcal{S}_{t}}$ identifies a team with workers of skill levels $\mathcal{S}_{t}$, and

$$
V\left(t^{\mathcal{S}_{t}} \mid P A M\right)=\int_{0}^{1} h(y) d F^{P A M, \mathcal{S}_{t}}(y)
$$

indicates the expected payoff of team $t^{\mathcal{S}_{t}}$, assuming that all other workers are matched positively assorted. As in the previous sections, $F^{P A M, \mathcal{S}_{t}}(y)$ is the rank distribution of a team $t$ with skill levels $\mathcal{S}_{t}$ when competing against teams that are arranged according to PAM, and $f^{P A M, \mathcal{S}_{t}}(y):=\frac{d F^{P A M, \mathcal{S}_{t}}}{d y}(y)$ is the associated PDF. ${ }^{26}$ It can be verified that wages in an equilibrium with positive assortative matching satisfy

$$
w^{s}=\frac{1}{N} \cdot V\left(t^{\{s\}^{N}} \mid P A M\right), \text { for all } s \in \mathcal{S} .
$$

Using the above equilibrium wages and rearranging terms, we obtain that there is an equilibrium with positive assortative matching if and only if

$$
\frac{1}{N} \cdot \sum_{s \in \mathcal{S}_{t}} V\left(t^{\{s\}^{N}} \mid P A M\right) \geq V\left(t^{\mathcal{S}_{t}} \mid P A M\right), \text { for all } \mathcal{S}_{t} \in \mathcal{S}^{N} .
$$

This is again a supermodularity condition.

Now, consider the following adaptation of Assumption 1(i):

\section{Assumption 1'}

For any multiset of skills $\mathcal{S}_{t} \in \mathcal{S}^{N}$, it holds that

$$
\mathcal{A}:=\left\{y \in[0,1]: f^{P A M, \mathcal{S}_{t}}(y) \geq \frac{1}{N} \cdot \sum_{s \in \mathcal{S}_{t}} f^{P A M,\{s\}^{N}}(y)\right\}
$$

is a convex and compact set.

Analogously to the case of two types and two team members, the most natural interpretation of Condition (25) is that the rank distribution of a mixed team is biased towards achieving mid-range ranks when compared to assortatively matched teams of the types corresponding to its team members. Now, for a given $\mathcal{S}_{t} \in \mathcal{S}^{N}$, we can rewrite Condition (24) as

$$
\int_{0}^{1} h(y) \cdot \frac{1}{N} \cdot \sum_{s \in \mathcal{S}_{t}} f^{P A M,\{s\}^{N}}(y) d y \geq \int_{0}^{1} h(y) \cdot f^{P A M, \mathcal{S}_{t}}(y) d y
$$

and we can then apply Lemma 1 from Appendix A to generalize Corollary 1 to the case with $S \geq 2$ types and $N \geq 2$ team members.

\section{Corollary 3}

Let Assumption $1^{\prime}$ be satisfied. Then Corollary 1 extends to the case with $S \geq 2$ types and $N \geq 2$ team members.

\footnotetext{
${ }^{26}$ We assume that for all $\mathcal{S}_{t} \in \mathcal{S}^{N}, f^{P A M}, \mathcal{S}_{t}$ satisfies the regularity conditions imposed on $f^{\alpha, k}$ in Section 4 .
} 


\subsection{Beyond rank competition}

Thus far we have considered economies with competition for rank. In such cases, there is a clear distinction between rank-dependent payoffs that are affected by globalization, on the one hand, and matching outcomes and the competition that impact teams' rank distributions, on the other. The focus on rank competitions therefore allows us to discuss our main mechanisms of interest in a transparent way. Yet, as we show next, our main results also apply to models where a team's payoff directly depends on its own productivity and the productivities of all other teams in the economy, which greatly expands the application range of our results.

Let us assume that each team forms and then receives a random productivity draw $\varphi$ from a publicly-known, skill-dependent probability distribution $B^{k}(\varphi), k \in\{l, m, h\}$, that has support $\Phi \subseteq \mathbb{R}_{+}$, with density function $b^{k}(\varphi):=\frac{d B^{k}(\varphi)}{d \varphi}$. After receiving their productivity draw, teams compete in a market where each team's payoff depends on its own productivity and the productivities of all other teams in the economy, analogously to e.g. a Melitz (2003)-model or models with monopolistic competition more generally (see Section 8.2.2 for a discussion). These productivities of the competing teams depend on the (whole) matching. With a continuum of workers, however, there is no aggregate uncertainty about the distribution of productivities in the economy, once the matching is given. Hence, we can summarize the entire distribution of productivities in the economy by parameter $\alpha$, which denotes the share of teams whose members are matched positively assorted. Accordingly, we use $\pi^{\alpha}(\varphi)$, with $\frac{d \pi^{\alpha}(\varphi)}{d \varphi} \geq 0$, to denote the payoff of a team with productivity $\varphi$ given matching $\alpha$ (and the ensuing productivity distribution of all teams in the economy). Then, the expected payoff of a team $k \in\{l, m, h\}$ given PAM is

$$
V\left(t^{k} \mid \mu(1)\right)=\int_{\varphi \in \Phi} \pi^{1}(\varphi) d B^{k}(\varphi)
$$

and PAM is an equilibrium if and only if

$$
\int_{\varphi \in \Phi} \pi^{1}(\varphi) d B^{m}(\varphi) \leq \int_{\varphi \in \Phi} \pi^{1}(\varphi) \cdot \frac{1}{2} \cdot\left[d B^{l}(\varphi)+d B^{h}(\varphi)\right] .
$$

Now, consider the following adaptation of Assumption 1:

\section{Assumption $1^{\prime \prime}$}

The set

$$
\mathcal{A}:=\left\{\varphi \in \Phi: b^{m}(\varphi) \geq \frac{1}{2}\left[b^{l}(\varphi)+b^{h}(\varphi)\right]\right\}
$$

is convex and compact. 
Then we can again use Lemma 1 from Appendix A to show that Corollary 1 generalizes to this case for any form of globalization $g(\cdot)$ that is an increasing, convex transformation of payoffs conditional on $\operatorname{PAM}, \pi^{1}(\varphi) .{ }^{27}$

\section{Corollary 4}

Let Assumption 1" be satisfied and suppose that globalization is an increasing, convex transformation of payoffs conditional on $\operatorname{PAM}, \pi^{1}(\varphi)$. Then Corollary 1 extends to the case with productivity dependent payoffs.

\subsection{A necessary and sufficient restriction on the competition}

Our results are centered on Assumption 1, which in essence requires that mixed teams are relatively more likely to achieve mid-range ranks (or mid-range productivities in case of Section 7.2). This assumption can be relaxed further. This is because Lemma 1 in Appendix A, which is central for the derivation of our main results, relies on the following assumption:

\section{Assumption $1^{\prime \prime \prime}$}

$F^{\alpha, m}$ crosses $\frac{1}{2} \cdot\left[F^{\alpha, l}+F^{\alpha, h}\right]$ at most once and if it does, it does it from below.

It is straightforward to verify that the single-crossing condition in Assumption $1^{\prime \prime \prime}$ is a strict generalization of Assumption 1 (recall Equation (5)). Nevertheless, all our results hold with this weaker assumption as well. While Assumption 1 is naturally satisfied in many different contexts - see Section 8 - and it allows to build intuition for the economic content, the weaker version in Assumption 1"' may nevertheless come in handy for certain applications, e.g. when considering skill-dependent payoffs and discrete distributions for teams' skills as in Chade and Eeckhout (2020, Section 5.1). We revisit this point in Section 8.3.

Finally, it is worth noting that Assumption 1 cannot be relaxed further without loss of generality in terms of admissible payoff schemes. Athey (2001, Proposition 2(ii)) shows that the single-crossing condition in Assumption $1^{\prime \prime \prime}$ is necessary and sufficient for our main result to hold for all $h \in \mathcal{H}$ and $g \in \mathcal{G}$.

\subsection{Core allocations}

For the definition of equilibrium we have relied on a standard notion of pairwise stability. Without externalities, requiring that no two workers want to dissolve their respective teams

\footnotetext{
${ }^{27}$ To simplify the presentation, in Lemma 1 (see Appendix A) we have considered distributions on $[0,1]$. Note, however, that this restriction is not necessary, and we can allow for distributions with unbounded support, for example - see also Section 7.3.
} 
and form a new pair - as is done in Definition 1 - is equivalent to requiring that no coalition of workers of any size (measure) wants to do so. In the presence of externalities at the matching stage, however, a subset of workers that deviates and match differently can affect their expected payoffs not only due to their having new partners (direct effect), but also because they create spillover effects on the entire market (indirect externality effect) by changing the share of teams that are matched positively assorted. Nevertheless, our analysis can also be applied to core allocations where we allow coalitions of workers to jointly deviate. See Appendix C.2 for further details.

\subsection{Migration}

In our analysis, we have taken the pool of workers as given and, in particular, we have assumed that it is itself not affected by globalization. While in many instances this may be a reasonable approximation given that capital, goods, and services tend to be more mobile than people, in other cases - such as our illustrative example from European footballworkers are relatively mobile as well. It is thus relevant to discuss how our results are affected by labor mobility. To that end, we consider two polar cases.

First, assume that there are two identical economies that were initially separated and then integrated their labor markets, but which still have separate competitions. In the case of European football, for example, teams still compete in their national leagues. Our results remain valid in this first polar approach to labor mobility. That is, globalization in the form of a convex transformation of payoffs increases the concentration of talent with this extreme form of labor mobility as well. This is because with perfectly mobile labor, wages for highand low-skilled workers have to be the same across countries. Hence, migration will not change the skill composition of the two economies. Referring to our football example, it is indeed not the case that the very best players all play in one country.

Our second polar case regarding labor mobility assumes that the two countries integrate completely, i.e. both their competition and their labor markets are merged. This corresponds to a simple scaling of our economy, and our results directly apply as long as the competition and the payoff structure are scale invariant. ${ }^{28}$

\footnotetext{
${ }^{28}$ In this case, integrating the economies yields a payoff scheme $\tilde{h}(y)$, with $\tilde{h}(2 y):=h(y)$, and a rank distribution for a team $k \in\{l, m, h\}$ in an economy where a share $\alpha$ of teams have members who are matched positively assorted $\tilde{f}^{\alpha, k}(y)$, where $\tilde{f}^{\alpha, k}(2 y):=f^{\alpha, k}(y)$. A simple change of variables $x:=2 y$ then implies that integration will not impact equilibrium outcomes.
} 


\section{Micro-foundations and Applications}

In the previous sections, we have presented a simple reduced-form analysis of how globalization impacts the concentration of talent across competing teams. In this section, we present micro-foundations for our two main assumptions: Assumption 1 (about competition) and Assumption 3 (about globalization). We begin with the former, which specifically refers to how the competition mode determines the ranking, and then consider the latter, which specifically refers to how globalization impacts payoffs. We finally show that our results apply to other contexts as well by briefly discussing a selection of papers from different fields that consider amplified superstar effects.

\subsection{Micro-foundations for the competition mode}

\subsubsection{Head-to-head competition}

Suppose that all teams compete head to head and are then ranked according to the number of victories. ${ }^{29}$ This is in line with competition in a sports league, for example. More specifically, assume that each time two teams meet, there is a fixed probability $p>\frac{1}{2}$ that the higher skilled team wins, with that probability being $\frac{1}{2}$ if two equally skilled teams compete. Then, as the number of games that a particular team is involved in goes to infinity, the ratio of victories for this team will converge to its expected value by the law of large numbers. ${ }^{30}$ As a consequence, the rank distribution of a low-skilled team will be uniform on $\left[0, \frac{\alpha}{2}\right]$, that of a mixed team will be uniform on $\left[\frac{\alpha}{2}, 1-\frac{\alpha}{2}\right]$ and that of a high-skilled team uniform on $\left[1-\frac{\alpha}{2}, 1\right] .{ }^{31}$ This implies that the set $\mathcal{A}_{\alpha}^{\mathcal{F}}$ is (asymptotically) convex and compact, in which case Assumption 1 is satisfied.

\subsubsection{Patent race}

Assumption 1 also arises naturally when teams compete against each other in a patent or innovation race. To show this, assume that after teams have formed, they are ranked according to the timing of first events drawn from a Poisson process with skill-dependent

\footnotetext{
${ }^{29}$ See also Section 3.

${ }^{30} \mathrm{We}$ follow the convention in the economics literature and apply the law of large numbers to a continuum of random variables.

${ }^{31}$ To see this, note that the expected share of victories for a low-skilled team is $p^{l}=\frac{\alpha}{4}+\left(1-\frac{\alpha}{2}\right) \cdot(1-p)$, that of a mixed team is $p^{m}=\frac{\alpha}{2} \cdot p+\frac{\alpha}{2} \cdot(1-p)+\frac{1-\alpha}{2}=\frac{1}{2}$, and that of a high-skilled team is $p^{h}=\frac{\alpha}{4}+\left(1-\frac{\alpha}{2}\right) \cdot p$. It is then straightforward to verify that for every $p>\frac{1}{2}$, we must have $p^{l}<p^{m}<p^{h}$. The resulting rank CDFs are not continuously differentiable. Note, however, that this is not necessary for our results - see Section 7.3. Moreover, we could easily generalize this toy example by assuming that the overall skill level of a team is itself subject to a random shock such that some teams of low-skilled workers, for example, end up being relatively high skilled.
} 
arrival rate $\lambda^{k}>0$, with $k \in\{l, m, h\}$. That is, the cumulative distribution function for the time of invention of a team with skill level $\lambda^{k}$ is ${ }^{32}$

$$
B^{k}(z)=1-e^{-\lambda^{k} z}
$$

As usual, we let $b^{k}(z)$ denote the corresponding PDF. In an economy with a share $\alpha$ of all teams whose members are matched positively assorted, the (expected) rank $y \in[0,1]$ of a team that invents at some time $z \in \mathbb{R}_{+}$is then given by

$$
y=r^{\alpha}(z)=\frac{\alpha}{2} e^{-\lambda^{l} z}+\frac{\alpha}{2} e^{-\lambda^{h} z}+(1-\alpha) e^{-\lambda^{m} z},
$$

where $(\alpha / 2) e^{-\lambda^{l} z}$ is the measure of low-skilled teams that have not yet innovated at time $z$ and analogously for $(\alpha / 2) e^{-\lambda^{h} z}$ and $(1-\alpha) e^{-\lambda^{m} z}$. As we show in Appendix C.3, the rank distribution of the mixed team satisfies Assumption 1 for any $\lambda^{l}, \lambda^{m}, \lambda^{h}>0$. Of course, if skills are to be meaningful, it should be the case that $\lambda^{l} \leq \lambda^{m} \leq \lambda^{h}$.

\subsubsection{Pareto distribution of team's productivity}

Finally, we show that Assumption $1^{\prime \prime}$ is satisfied if a team's productivity is drawn from a Pareto distribution with skill-dependent location parameter. More specifically, given $\gamma>0$ let

$$
b^{k}(\varphi):= \begin{cases}\frac{\gamma \varphi^{k \gamma}}{\varphi^{\gamma+1}} & \text { if } \varphi \geq \underline{\varphi}^{k} \\ 0 & \text { otherwise }\end{cases}
$$

be the PDF for a team with skill level $k \in\{l, m, h\}$, and then suppose that $\underline{\varphi}^{l} \leq \underline{\varphi}^{m} \leq \underline{\varphi}^{h}$. It immediately follows that the set

$$
\mathcal{A}:=\left\{\varphi \in \Phi: b^{m}(\varphi) \geq \frac{1}{2}\left[b^{l}(\varphi)+b^{h}(\varphi)\right]\right\}
$$

is convex. Hence, Assumption $1^{\prime \prime}$ is satisfied..$^{33}$

\subsection{Micro-foundations for globalization as convex transformation}

To rationalize Assumption 3, we build on the models of Rosen (1981) and Melitz (2003), respectively.

\footnotetext{
${ }^{32}$ See Loury (1979) and Dasgupta and Stiglitz (1980) for seminal contributions using Poisson processes in the modeling of patent races.

${ }^{33}$ If $\underline{\varphi}^{m \gamma} \geq \frac{1}{2}\left[\underline{\varphi}^{l \gamma}+\underline{\varphi}^{h \gamma}\right]$, we have $\mathcal{A}=\left[\underline{\varphi}^{m}, \infty\right)$. If $\underline{\varphi}^{m \gamma}<\frac{1}{2}\left[\underline{\varphi}^{l \gamma}+\underline{\varphi}^{h \gamma}\right]$, we have $\mathcal{A}=\left[\underline{\varphi}^{m}, \underline{\varphi}^{h}\right)$. These sets are not compact. This, however, is not necessary for our results-see Section 7.3.
} 


\subsubsection{Rosen (1981) with competing teams}

In his seminal paper, Rosen (1981) shows how small differences in talent can result in large differences in sales and income at the top in the case of markets with imperfect substitutability of quantity for quality. Moreover, he shows that globalization-namely, an increase in the size of the market - results in a convex transformation of payoffs. In this section, we briefly discuss a simple variant of his model with competing teams and show how it maps into our reduced-form analysis.

Suppose that consumers demand overall services $x:=n z$ from competing teams, where $z$ is the quality of service provided by a team and $n$ is the quantity consumed, such as the number of games attended. Consumers face a fixed cost $s$ per unit of service consumed, which we can think of as representing e.g. the time cost associated with consuming the service. Hence, the total cost of consuming $n$ units of a service with quality $z$ is equal to $n(p(z)+s)$, where $p(z)$ is the price per unit of service of quality $z$. The cost per effective unit is $v:=(p(z)+s) / z$. The latter cost is constant across teams in equilibrium. Rosen (1981) allows for the existence of internal and external dis-economies of scale, i.e. the cost of providing $m$ units of service, $C(m)$, is increasing and convex. In addition, the quality of service $z=z(y, m)$ is decreasing in the number of units sold, reflecting congestion effects. ${ }^{34}$ In this setup, $y$ is a measure of the underlying value of the service provided by a team, and we can allow for different interpretations of $y$. In what follows, we think of $y$ simply as the rank of the team - reflecting the fact that supporters enjoy seeing their team winning - but it could also express the skill level of the team drawn from a random distribution as discussed in Section 7.2. Rosen (1981) shows that the payoff of a team ranked $y, h(y)$, satisfies

$$
h^{\prime \prime}(y)=v \cdot\left(z_{y}+m \cdot z_{y m}\right) \frac{\partial m}{\partial y}+v \cdot m \cdot z_{y y},
$$

i.e. the revenue function is convex over ranks if $z_{y y} \geq 0$ and $z_{y m} \geq 0$, where the latter inequality implies that higher ranked teams are better at serving larger audiences. The intuition is that in this case higher-ranked teams cannot only charge a higher price, but it is also profitable for them to serve larger audiences, which implies a convex payoff-scheme. The important point to note is that convexity increases in $v$, which is the market price per unit of service. That is, when in the wave of globalization demand for these services goes up so that $v$ increases, the payoff scheme becomes more convex, i.e. Assumption 3 holds. Hence, our reduced-form analysis applies to this variant of the Rosen (1981) model, provided that the competition satisfies Assumption 1.

\footnotetext{
${ }^{34}$ For example, Rosen (1981) suggests that it is more valuable to attend a concert in a small concert hall than in the Yankee Stadium.
} 


\subsubsection{Fixed cost of market entry: Melitz (2003)-model with entrepreneurial teams}

Assumption 3 is also naturally satisfied if teams have the opportunity to access foreign markets, albeit at a fixed cost $\kappa \geq 0$. Suppose that the gains from entering a foreign market are increasing with a team's rank in its domestic economy. In the case of European football, we may think of entering a foreign market as actively trying to increase a team's fan base in this market to raise revenues via sponsoring, merchandising, or licensing, for example. Such endeavors are naturally more promising for teams that perform well in their domestic leagues. ${ }^{35}$ Now, assume for simplicity that payoffs generated abroad, $\tilde{h}(y)$, are proportional to the domestic payoffs $h(y)$, i.e.

$$
\tilde{h}(y)=\lambda \cdot h(y)
$$

for some constant $\lambda>0$. Teams will enter the foreign market only if this is profitable, implying that the total payoff of a team ranked $y$ is

$$
g(h(y))=h(y)+\max \{0, \lambda \cdot h(y)-\kappa\}
$$

It is straightforward to verify that $g(\cdot)$ is increasing and convex..$^{36,37}$

The same logic also implies that our analysis directly applies to a simple variant of a Melitz (2003)-model with entrepreneurial teams. In this variant, workers are either high-skilled or low-skilled. High-skilled workers are better entrepreneurs, but they have no advantage when employed as a worker. There is an initial stage where workers decide whether or not to become an entrepreneur. Entrepreneurs match to form entrepreneurial teams of two and found a firm. As in the workhorse version of a Melitz (2003)-model, each firm is equipped with a distinct variety, and receives a random productivity draw, $\varphi$, from a known Pareto distribution. In this variant, however, the minimum value of $\varphi$ is increasing in the skill level of the entrepreneurial team. Firms with productivity draws above some endogenous threshold level start operating, while all other firms exit immediately, as in the

\footnotetext{
${ }^{35}$ In Germany, for example, Bayern Munich and Borussia Dortmund, the biggest and most successful football clubs in recent years, are most actively promoting their teams abroad and they are the only clubs running foreign offices (see https://www.welt.de/sport/article157261763/Das-Millionenspiel-der-Bundesligaklubsin-Uebersee.html). They also have by far the most facebook likes outside of Germany (see http://meedia.de/ 2015/09/23/bundesliga-bis-3-liga-das-grosse-facebook-ranking-der-fussballclubs/, retrieved on 10 September 2020).

${ }^{36}$ In this example $g(h(\cdot))$ is not differentiable for all $y \in[0,1]$. Nevertheless, our results do not hinge crucially on this regularity assumption, which we have only imposed for simplicity.

${ }^{37}$ We consider the case where globalization gives rise to a convex transformation of teams' payoffs directly. Alternatively, globalization may yield a convex transformation of firm sizes and then spill over to the compensation of managerial teams. Gabaix and Landier (2008), for example, present a model where at the top CEO pay (executive board pay in a simple variant with managerial teams) is proportionate to a power function of firm size, i.e. for all power coefficients larger than or equal to one, a globalization-induced convex transformation of firm sizes with $g(0)=0$ translates into a convex transformation of executive compensation.
} 
canonical Melitz (2003)-model. This implies that under autarky, a firm's profit is a piecewise linear and convex function of $\varphi^{\sigma-1}$, where $\sigma>1$ is the constant elasticity of substitution between varieties. The most interesting case is one with selection into exporting, in line with empirical facts. Globalization - a move from autarky to an equilibrium with tradethen implies that the minimum-productivity threshold for firms increases, i.e. the lowest productive firms are forced to exit after a trade liberalization. Firms with intermediate levels of productivity only serve their domestic market, and the most productive firms also export. The key point is that globalization gives rise to a piecewise linear and convex transformation of a firm's profits as a function of $\varphi^{\sigma-1}$ — we refer to Melitz and Redding (2014) for further details. Moreover, $\varphi^{\sigma-1}$ is Pareto distributed as well, and mixed teams are thus relatively more likely to have mid-range values of $\varphi^{\sigma-1}$, as shown in Section 8.1.3. This implies that this variant of the Melitz (2003)-model reduces to the model analyzed in Section 7.2 and, hence, our work points to an alternative channel through which trade may impact aggregate productivity and welfare: the composition of entrepreneurial teams. We refer to Appendix C.4 for further discussions. ${ }^{38}$

\subsection{Other applications}

So far, we have focused on globalization as a source of amplified superstar effects. While globalization is a prime example of the mechanisms we are interested in, 'superstar effects' can arise for various reasons, and alternative mechanisms have been proposed in the literature. In this section we briefly discuss some examples from different fields. The key point is that when introduced in environments with competing teams as considered here, each of these examples gives rise to an increased concentration of talent, no matter the exact underlying driver of an amplified superstar effect.

\subsection{1 (Skill-biased) technological change}

We begin with considering technological change, which has been identified as a driver of a higher segregation of the labor force by Kremer and Maskin (1996) and Acemoglu (1999). Kremer and Maskin (1996) consider a matching model where complementary tasks differ in their skill intensities. They then show how a higher skill-dispersion-which may arise from skill-biased technological change - can lead to more segregation of the labor force. Acemoglu

\footnotetext{
${ }^{38}$ In the Melitz (2003)-model, the total mass of entering firms is endogenous, which impacts the distribution of skills over entrepreneurial teams. In turn, this mass of entering firms depends on the matching. With a Pareto distribution of firm productivities, however, it does not directly depend on the trade environment. Hence, the endogeneity of the mass of entering firms does not impede the applicability of our main results, as these depend only on the redistributive effects of globalization conditional on the matching outcome. See Appendix C.4 for further details.
} 
(1999) presents a model where skill-biased technological change induces firms to create jobs that are skill-specific. In the following we present a complementary channel through which skill-biased technological change impacts the composition of teams: amplified superstar effects.

It is well known that skill-biased technological change or investment in skill-augmenting capital ceteris paribus increases the wage gap between high-skilled and low-skilled workers (see, e.g. Krusell et al. 2000; Card and DiNardo 2002). While in itself this does not imply an amplified superstar effect (Jones and Kim, 2018), technological change results in a convex transformation of payoffs if the complementarity between skills and technology is sufficiently strong. This is, for example, the case for the 'skill-biased change in entrepreneurial technology' considered in Poschke (2018). ${ }^{39}$

Another possibility is that technological change directly affects the complementarity between team members' skills. As shown in Chade and Eeckhout (2020, Section 5.1), this can lead to an increase in the share of teams that are positively assortatively matched in equilibrium. Interestingly though, Chade and Eeckhout (2020) restrict the parameter space in a way such that technological change results in a convex transformation of payoffs. Hence, their (parametric) result is a reflection of the much more general logic we put forward - see Appendix C.5 for further details.

\subsubsection{Urban agglomeration}

Our work may also help explaining why assortative matching is stronger in larger cities (see e.g. Andersson et al., 2007; Dauth et al., 2016). ${ }^{40}$ On the one hand, larger cities provide larger markets and this may amplify superstar effects as shown in Rosen (1981) and discussed in Section 8.2. On the other hand, it has been empirically documented that the skill premium increases with city size (Wheeler, 2001; Baum-Snow and Pavan, 2012). To rationalize this pattern, Behrens and Robert-Nicoud (2015) assume that there is a complementarity between the city size and the skill level of a worker. ${ }^{41}$ Most importantly for our purposes, they also consider a case where the complementarity is sufficiently strong such that the income scheme

\footnotetext{
${ }^{39}$ Specifically, in the model by Poschke (2018) a higher aggregate technology gives rise to a convex transformation of the profits of (potential) entrepreneurs as a function of their ability. Manasse and Turrini (2001) consider a skill-biased entrepreneurial technology in the context of a two-country Krugman (1980) trade model where heterogeneous entrepreneurs manage firms and where the entrepreneur's skill level determines the productivity - output quality in their case - of the firm. They make the point that 'superstar effects' arise if the productivity of a firm is elastic in the skill level of the entrepreneur, but they do not consider comparative statics with respect to this elasticity.

${ }^{40}$ Strictly speaking, these papers consider matching of workers to firms. Yet, if in larger cities higher skilled workers are more systematically matched to highly productive firms, then this also implies that they are more similar in terms of their skill levels to their co-workers, which is our focus here.

${ }^{41}$ Davis and Dingel (2019) motivate this assumption by arguing that higher skilled workers are better at drawing from the pool of knowledge that cities provide.
} 
is more convex in larger cities - see Behrens and Robert-Nicoud (2015, Section 4.5.2).

\subsubsection{Inter-occupational spillovers}

Gottlieb et al. (2019) propose yet another source of amplified superstar effects: interoccupational spillovers. In particular, they present a model where high-skilled specialistsdoctors, for example, or teams of lawyers in a set-up with competing teams - provide indivisible services to generalists. They then show that at the very top the income of specialists is a power function of their ability, and where the exponent is inversely related to the Pareto-shape parameter of the income distribution of generalists. Hence, an increase in (top-)income inequality for 'generalists' implies a convex transform of payoffs for specialists at the top. ${ }^{42}$

\subsubsection{Taxation}

Lastly, we briefly discuss to what extent more progressive taxes could be used to mitigate superstar effects and, as a result, reduce the concentration of talent in an economy. ${ }^{43}$ Whenever taxes are collected at the team level, our previous analyses readily apply and a convex transformation of the tax schedule makes PAM less likely. ${ }^{44}$ In many instances, however, taxation occurs at the individual level, and in such cases additional effects have to be taken into consideration. A detailed account of these effects is beyond the scope of our paper, but our work nevertheless allows identifying in simple terms the key trade-off.

In our model, teams first form and then compete against each other with uncertain outcomes. This means that the wages of high- and low-skilled workers refer to their respective expected incomes, but their actual incomes differ according to the realized outcomes in the competition stage. Because workers are risk-neutral, this did not matter for our analysis so far. When it comes to taxation, however, the fact that the ex-post payoffs are different from the ex-ante expected payoffs does matter. It implies that a convex transform of the tax schedule has two opposing effects on the matching. On the one hand, it implies a higher average tax burden for positively assorted teams as these have - on average - a higher prob-

\footnotetext{
${ }^{42}$ Jones and Kim (2018), for example, show how the Pareto shape parameter of the income distribution of entrepreneurs changes with the rate of creative destruction and the cost of effort.

${ }^{43}$ In this vein, our paper is also, but less closely, related to the literature analyzing optimal taxation in matching markets - see, e.g. Konrad and Lommerud (2010) Scheuer and Werning (2017).

${ }^{44}$ Several definitions of tax progressivity are used in the literature and, in general, they neither imply nor are implied by a convex transformation of the tax schedule. Nevertheless, an increasing and convex transformation of the tax schedule is an important case of more progressive taxes and in many instances it overlaps with alternative definitions. For example, one can easily verify that the net income under a flatrate tax schedule is a convex transformation of the net income under a two-rate tax schedule that preserves aggregate net incomes. We note that the latter tax schedule is more progressive than the former according to average tax progression (Musgrave and Thin, 1948).
} 
ability of achieving the highest ranks, i.e. those that are affected most by the change in the tax scheme. This is the counterpart of the mechanism that we scrutinize in this paper. There is, however, a second effect at play: Positively assorted teams split the team income more equally among themselves when compared to negatively assorted teams, and the more convex the tax scheme, the more harmful is an unequal split. Whether or not a more progressive tax scheme results in less positive assortative matching then depends on which effect dominates. In other words, it is not necessarily the case that more progressive taxes contribute to a lower concentration of talent. Further details are provided in Appendix C.6.

\section{Conclusion}

We have investigated the effect of a convex transformation of payoffs on the concentration of talent in large matching markets. We have argued that globalization can attest for this type of transformation of the payoff scheme. This implies that in relative terms high ranks are rewarded higher prizes after globalization than before. We have chosen a reduced-form approach to modelling competition that rests on minimal assumptions pertaining to the relationship between skills and market outcomes, and we expect the mechanisms we have considered to be relevant for many actual markets with competing teams. Because payoffs in such markets might be - and often are - influenced by exogenous elements (namely, globalization), our research question clearly seems relevant both theoretically and empirically.

Our main insight is that globalization promotes the concentration of talent, i.e., it may result in the emergence of more positive assortative matching. This has important distributional consequences, as it feeds back into the income distribution of modern societies. Moreover, potential adverse effects of globalization over and above a direct effect on income inequality have received growing attention in recent years (Autor et al., 2014; Che et al., 2016; McManus and Schaur, 2016; Pierce and Schott, 2020). An increased concentration of talent might be an important factor in this regard, because in the presence of learning externalities it may harm low-skilled workers and perpetuate - or even increase - skill differences. More generally, a greater concentration of talent contributes to social segregation. Future work may set out to study such effects and their welfare implications in more detail.

While we have focused on globalization as an amplifier of superstar effects, such effects can arise for various reasons, and the literature has ascribed them to technological change, urban agglomeration, and spillover-effects between buyers and sellers, for example. Our analysis holds regardless of the exact source of amplified superstar effects and, hence, our work reveals a general and robust driver of an increased concentration of talent in an economy. 


\section{References}

Acemoglu, D. (1999). Changes in unemployment and wage inequality: An alternative theory and some evidence. American Economic Review, 89(5):1259-1278.

Andersson, F., Burgess, S., and Lane, J. I. (2007). Cities, matching and the productivity gains of agglomeration. Journal of Urban Economics, 61(1):112-128.

Arrow, K. J. (1971). Essays in the theory of risk-bearing.

Athey, S. (2001). Monotone comparative statics under uncertainty. Quarterly Journal of Economics, 117(1).

Autor, D. H., Dorn, D., and Hanson, G. H. (2013). The China syndrome: Local labor market effects of import competition in the United States. American Economic Review, 103(6):2121-2168.

Autor, D. H., Dorn, D., Hanson, G. H., and Song, J. (2014). Trade adjustment: Worker-level evidence. Quarterly Journal of Economics, 129(4):1799-1860.

Baum-Snow, N. and Pavan, R. (2012). Understanding the city size wage gap. Review of Economic Studies, 79(1):88-127.

Becker, G. S. (1973). A theory of marriage: Part I. Journal of Political Economy, 81(4):813846.

Behrens, K. and Robert-Nicoud, F. (2015). Agglomeration theory with heterogeneous agents. In Duranton, G., Henderson, J. V., and Strange, W. C., editors, Handbook of Regional and Urban Economics, volume 5. Elsevier.

Bénabou, R. and Tirole, J. (2016). Bonus culture: Competitive pay, screening, and multitasking. Journal of Political Economy, 124(2):305-370.

Bernard, A. B., Jensen, J. B., Redding, S. J., and Schott, P. K. (2007). Firms in international trade. Journal of Economic Perspectives, 21(3):105-130.

Card, D. and DiNardo, J. E. (2002). Skill-biased technological change and rising wage inequality: Some problems and puzzles. Journal of Labor Economics, 20(4).

Card, D., Heining, J., and Kline, P. (2013). Workplace heterogeneity and the rise of West German wage inequality. Quarterly Journal of Economics, 128(3):967-1015. 
Chade, H. and Eeckhout, J. (2020). Competing teams. Review of Economic Studies, 87(3):1134-1173.

Che, Y., Lu, Y., Pierce, J. R., Schott, P. K., and Tao, Z. (2016). Does Trade Liberalization with China Influence U.S. Elections? Working Paper 22178, NBER.

Chung, K.-S. (2000). On the existence of stable roommate matchings. Games and Economic Behavior, 33(2):206-230.

Costinot, A. and Vogel, J. (2010). Matching and inequality in the world economy. Journal of Political Economy, 118(4):747-786.

Dasgupta, P. and Stiglitz, J. (1980). Industrial structure and the nature of innovative activity. Economic Journal, 90(358):266-293.

Dauth, W., Findeisen, S., Moretti, E., and Suedekum, J. (2016). Spatial wage disparitiesworkers, firms, and assortative matching. Technical report, Heinrich Heine University Düsseldorf.

Dauth, W., Findeisen, S., and Suedekum, J. (2014). The rise of the east and the far east: German labor markets and trade integration. Journal of the European Economic Association, 12(6):1643-1675.

Davis, D. R. and Dingel, J. I. (2019). A spatial knowledge economy. American Economic Review, 109(1):153-170.

Diamond, P. A., Stiglitz, J. E., et al. (1974). Increases in risk and in risk aversion. Journal of Economic Theory, 8(3):337-360.

Dix-Carneiro, R. and Kovak, B. K. (2017). Trade reform and regional dynamics. American Economic Review, 107(10):2908-2946.

Eeckhout, J. and Kircher, P. (2018). Assortative matching with large firms. Econometrica, 86(1):85-132.

Faber, B. (2014). Trade Liberalization, the Price of Quality, and Inequality: Evidence from Mexican Store Prices. Mimeo.

Fajgelbaum, P. D. and Khandelwal, A. K. (2016). Measuring the unequal gains from trade. Quarterly Journal of Economics, 131(3):1113-1180.

Gabaix, X. and Landier, A. (2008). Why has CEO pay increased so much? Quarterly Journal of Economics, 123(1):49-100. 
Gale, D. and Shapley, L. S. (1962). College admissions and the stability of marriage. American Mathematical Monthly, 69(1):9-15.

Galle, S., Rodríguez-Clare, A., and Moises, Y. (2017). Slicing the pie: Quantifying the aggregate and distributional effects of trade. Working Paper 23737, NBER.

Geerolf, F. (2017). A Theory of Pareto Distributions. Mimeo.

Gersbach, H. and Schmutzler, A. (2014). Does globalization create superstars? A simple theory of managerial wages. European Economic Review, 71:34-51.

Gottlieb, J. D., Hémous, D., Hicks, J., and Olsen, M. (2019). The Spillover Effects of Top Income Inequality. Mimeo.

Grossman, G. M., Helpman, E., and Kircher, P. (2016). Matching, sorting, and the distributional effects of international trade. Journal of Political Economy, 125(1):224-264.

Grossman, G. M. and Rossi-Hansberg, E. (2008). Trading tasks: A simple theory of offshoring. American Economic Review, 98(5):1978-1997.

Hafalir, I. E. (2008). Stability of marriage with externalities. International Journal of Game Theory, 37(3):353-369.

Hakanson, C., Lindqvist, E., and Vlachos, J. (2015). Firms and skills: The evolution of worker sorting. Working Paper 1072, Research Institute of Industrial Economics.

Hammond, J. S. I. (1974). Simplifying the choice between uncertain prospects where preference is nonlinear. Management Science, 20(7):1047-1072.

Haskel, J., Lawrence, R. Z., Leamer, E. E., and Slaughter, M. J. (2012). Globalization and US wages: Modifying classic theory to explain recent facts. Journal of Economic Perspectives, 26(2):119-40.

Helpman, E., Itskhoki, O., Muendler, M.-A., and Redding, S. J. (2017). Trade and inequality: From theory to estimation. Review of Economic Studies, 84(1):357-405.

Helpman, E., Itskhoki, O., and Redding, S. (2010). Inequality and unemployment in a global economy. Econometrica, 78(4):1239-1283.

Jewitt, I. (1989). Choosing between risky prospects: The characterization of comparative statics results, and location independent risk. Management Science, 35(1):60-70.

Jones, C. I. and Kim, J. (2018). A Schumpeterian model of top income inequality. Journal of Political Economy, 126(5):1785-1826. 
Karlin, S., Novikoff, A., et al. (1963). Generalized convex inequalities. Pacific Journal of Mathematics, 13(4):1251-1279.

Konrad, K. A. and Lommerud, K. E. (2010). Love and taxes-and matching institutions. Canadian Journal of Economics/Revue canadienne d'économique, 43(3):919-940.

Kremer, M. (1993). The O-ring theory of economic development. Quarterly Journal of Economics, 108(3):551-575.

Kremer, M. and Maskin, E. (1996). Wage inequality and segregation by skill. Working Paper 5718, NBER.

Kremer, M. and Maskin, E. (2006). Globalization and inequality. Mimeo, Harvard University.

Krugman, P. (1980). Scale economies, product differentiation, and the pattern of trade. American Economic Review, 70(5):950-959.

Krusell, P., Ohanian, L. E., Ríos-Rull, J.-V., and Violante, G. L. (2000). Capital-skill complementarity and inequality: A macroeconomic analysis. Econometrica, 68(5):10291053.

Lee, E. (2020). Trade, inequality, and the endogenous sorting of heterogeneous workers. Journal of International Economics, 125:103310.

Legros, P. and Newman, A. F. (2002). Monotone matching in perfect and imperfect worlds. Review of Economic Studies, 69(4):925-942.

Legros, P. and Newman, A. F. (2007). Beauty is a beast, frog is a prince: Assortative matching with nontransferabilities. Econometrica, 75(4):1073-1102.

Loury, G. C. (1979). Market structure and innovation. Quarterly Journal of Economics, 93(3):395-410.

Ma, L. and Ruzic, D. (2020). Globalization and top income shares. Journal of International Economics, 125:103312.

Määttänen, N. and Terviö, M. (2014). Income distribution and housing prices: An assignment model approach. Journal of Economic Theory, 151:381-410.

Manasse, P. and Turrini, A. (2001). Trade, wages, and superstars. Journal of International Economics, 54. 
Mas-Colell, A., Whinston, M. D., and Green, J. R. (1995). Microeconomic Theory. Oxford University Press, New York, NY.

Maskin, E. (2015). Why haven't global markets reduced inequality in emerging economies? World Bank Economic Review, 29:S48-S52.

McManus, T. C. and Schaur, G. (2016). The effects of import competition on worker health. Journal of International Economics, 102:160-172.

Melitz, M. J. (2003). The impact of trade on intra-industry reallocations and aggregate industry productivity. Econometrica, 71(6):1695-1725.

Melitz, M. J. and Redding, S. J. (2014). Chapter 1 - Heterogeneous Firms and Trade. In Gopinath, G., Helpman, E., and Rogoff, K., editors, Handbook of International Economics, volume 4. Elsevier.

Mumcu, A. and Saglam, I. (2010). Stable one-to-one matchings with externalities. Mathematical Social Sciences, 60(2):154-159.

Musgrave, R. A. and Thin, T. (1948). Income tax progression, 1929-48. Journal of political Economy, 56(6):498-514.

Neffke, F. (2019). The value of complementary co-workers. Science Advances, 5:eaax3370.

Pierce, J. R. and Schott, P. K. (2020). Trade liberalization and mortality: Evidence from us counties. American Economic Review: Insights, 2(1):47-64.

Piketty, T., Saez, E., and Stantcheva, S. (2014). Optimal taxation of top labor incomes: A tale of three elasticities. American Economic Journal: Economic Policy, 6(1):230-271.

Porzio, T. (2017). Cross-country differences in the optimal allocation of talent and technology. Mimeo.

Poschke, M. (2018). The firm size distribution across countries and skill-biased change in entrepreneurial technology. American Economic Journal: Macroeconomics, 10(3):1-41.

Pratt, J. W. (1964). Risk aversion in the small and in the large. Econometrica, 32(1/2):122136.

Pycia, M. and Yenmez, M. B. (2017). Matching with Externalities. Mimeo.

Rosen, S. (1981). The economics of superstars. American Economic Review, 71(5):845-858. 
Sasaki, H. and Toda, M. (1996). Two-sided matching problems with externalities. Journal of Economic Theory, 70(1):93-108.

Scheuer, F. and Werning, I. (2017). The taxation of superstars. Quarterly Journal of Economics, 132(1):211-270.

Shaked, M. and Shanthikumar, J. G. (2007). Stochastic orders. Springer Verlag, New York, NY.

Shimer, R. and Smith, L. (2000). Assortative matching and search. Econometrica, 68(2):343369.

Song, J., Price, D. J., Guvenen, F., Bloom, N., and von Wachter, T. (2019). Firming up inequality. Quarterly Journal of Economics, 134(1):1-50.

Terviö, M. (2008). The difference that CEOs make: An assignment model approach. American Economic Review, 98(3):642-68.

Wheeler, C. H. (2001). Search, sorting, and urban agglomeration. Journal of Labor Economics, 19(4):879-899. 


\section{Appendix}

\section{A Proof of Theorem 1}

Our goal is to show the following two inequalities:

$$
\underline{\alpha}_{h} \leq \underline{\alpha}_{g}
$$

and

$$
\bar{\alpha}_{h} \leq \bar{\alpha}_{g} .
$$

Under Assumption 4, we know from Proposition 2 that an equilibrium must exist with and without globalization. This means that $\underline{\alpha}_{h}, \underline{\alpha}_{g}, \bar{\alpha}_{h}, \bar{\alpha}_{g}$ are well defined and that, moreover, $\underline{\alpha}_{h}, \underline{\alpha}_{g}, \bar{\alpha}_{h}, \bar{\alpha}_{g} \in[0,1]$.

The following technical result is crucial for the proof and is implied by well-known results from the literature on decision under uncertainty (see Karlin et al., 1963; Hammond, 1974; Jewitt, 1989; Shaked and Shanthikumar, 2007).

\section{Lemma 1}

Let $h \in \mathcal{H}$ and $g \in \mathcal{G}$, and consider two continuously differentiable CDFs, $F(\cdot)$ and $\tilde{F}(\cdot)$, with support $[0,1]$. Then the following two statements hold:

(i) If

$$
\left\{y \in[0,1]: \frac{d F(y)}{d y} \leq \frac{d \tilde{F}(\cdot)}{d y}\right\}
$$

is a convex and compact set,

$$
\int_{0}^{1} g(h(y)) d \tilde{F}(y) \geq \int_{0}^{1} g(h(y)) d F(y) \Rightarrow \int_{0}^{1} h(y) d \tilde{F}(y) \geq \int_{0}^{1} h(y) d F(y) .
$$

(ii) If

$$
\left\{y \in[0,1]: \frac{d F(y)}{d y} \geq \frac{d \tilde{F}(y)}{d y}\right\}
$$

is a convex and compact set,

$$
\int_{0}^{1} h(y) d \tilde{F}(y) \geq \int_{0}^{1} h(y) d F(y) \Rightarrow \int_{0}^{1} g(h(y)) d \tilde{F}(y) \geq \int_{0}^{1} g(h(y)) d F(y) .
$$

For instance, Part (i) from Lemma 1 follows from Theorem 3 in Hammond (1974) - see his conditions (c2) and (d2). Part (ii) from Lemma 1 also follows from Theorem 3 in Hammond 
(1974) - see his conditions (c1) and (d1). Indeed, note that we can interpret $h(y)$ and $g(h(y))$ as two Bernoulli utility functions where $g(h(y))$ is less risk-averse in the Arrow-Pratt sense (Arrow, 1971; Pratt, 1964). Hence, it suffices to note that if

$$
\left\{y \in[0,1]: \frac{d F(y)}{d y} \leq \frac{d \tilde{F}(y)}{d y}\right\}
$$

is a convex and compact set, then either $F$ and $\tilde{F}$ dominate each other in the first stochastic order or $\tilde{F}$ crosses $F$ only once in $y \in(0,1)$ and it does it from below. ${ }^{45}$ Similarly, if

$$
\left\{y \in[0,1]: \frac{d F(y)}{d y} \geq \frac{d \tilde{F}(y)}{d y}\right\}
$$

is a convex and compact set, then either $F$ and $\tilde{F}$ dominate each other in the first stochastic order or $F$ crosses $\tilde{F}$ only once in $y \in(0,1)$ and it does it from below.

To see the use of this lemma for our purposes, note, for example, that by choosing $\tilde{F}(\cdot)$ to be the uniform distribution on $[0,1]$ and $F(\cdot)$ to be the rank distribution for a mixed team given PAM, the assumption in Lemma 1(ii) corresponds to Assumption 1 for the case of $\alpha=1$. Moreover, the two inequalities in Condition A.4 correspond to the necessary and sufficient condition for PAM to be an equilibrium before and after globalization, respectively.

We divide the remainder of the proof into three parts.

Part (i): Proof of Inequality (A.1)

We distinguish several cases with regard to the values of $\underline{\alpha}_{h}$ and $\underline{\alpha}_{g}$.

Case $I: \underline{\alpha}_{g}=1$

In this case, Inequality (A.1) holds trivially.

Case II: $\underline{\alpha}_{g}=0$

In this case, NAM is an equilibrium after globalization. Using Proposition 1, we obtain

$$
\begin{aligned}
\int_{0}^{1} g(h(y)) d y & \geq \int_{0}^{1} g(h(y)) \cdot\left(\frac{1}{2} d F^{\underline{\alpha}_{g}, h}(y)+\frac{1}{2} d F^{\underline{\alpha}_{g}, l}(y)\right) \\
& =\int_{0}^{1} g(h(y)) \cdot\left(\frac{1}{2} d F^{0, h}(y)+\frac{1}{2} d F^{0, l}(y)\right) .
\end{aligned}
$$

\footnotetext{
${ }^{45}$ Strictly speaking, the case where $F$ FOSD $\tilde{F}$ is not covered by Hammond (1974, Theorem 3 ). In such case, a well known result on FOSD (Mas-Colell et al., 1995, Proposition 6.D.1) immediately implies that

$$
\int_{0}^{1} h(y) d \tilde{F}(y) \leq \int_{0}^{1} h(y) d F(y), \text { for all } h \in \mathcal{H} .
$$

Moreover, it can be shown that whenever the condition holds with equality for $g(h(y))$, it must also hold with equality for $h(y)$.
} 
Then (A.3) (see Lemma 1) together with Assumption 1 implies that

$$
\int_{0}^{1} h(y) d y \geq \int_{0}^{1} h(y) \cdot\left(\frac{1}{2} d F^{0, h}(y)+\frac{1}{2} d F^{0, l}(y)\right)
$$

Hence, NAM is also an equilibrium before globalization, which means that $\underline{\alpha}_{h}=0$. That is, Inequality (A.1) holds.

Case III: $\underline{\alpha}_{g} \in(0,1)$

In this case, NAM is not an equilibrium after globalization, and there is an equilibrium (after globalization) with $\alpha<1$. Using Proposition 1, we obtain

$$
\int_{0}^{1} g(h(y)) d y=\int_{0}^{1} g(h(y)) d F^{\alpha_{g}, m}(y) .
$$

Due to (5), the above equality can be rewritten as

$$
\int_{0}^{1} g(h(y)) d y=\int_{0}^{1} g(h(y))\left[\frac{1}{2} d F^{\underline{\alpha}_{g}, l}(y)+\frac{1}{2} d F^{\underline{\alpha}_{g}, h}(y)\right] .
$$

Then (A.3) (see Lemma 1) together with Assumption 1 implies that

$$
\int_{0}^{1} h(y) d y \geq \int_{0}^{1} h(y)\left[\frac{1}{2} d F^{\alpha_{g}, l}(y)+\frac{1}{2} d F^{\alpha_{g}, h}(y)\right],
$$

which using (5) can be equivalently written as

$$
\int_{0}^{1} h(y) d y \leq \int_{0}^{1} h(y) d F^{\alpha_{g}, m}(y) .
$$

If (A.5) holds as equality, we are done since then $\underline{\alpha}_{g}$ is also an equilibrium before globalization, which means that Inequality (A.1) holds. Accordingly, consider that (A.5) holds strictly, or, equivalently, that

$$
\int_{0}^{1} h(y) d y>\int_{0}^{1} h(y)\left[\frac{1}{2} d F^{\underline{\alpha}_{g}, l}(y)+\frac{1}{2} d F^{\underline{\alpha}_{g}, h}(y)\right] .
$$

Then, by the continuity of $\int_{0}^{1} h(y) d F^{\alpha, l}(y)$ and $\int_{0}^{1} h(y) d F^{\alpha, h}(y)$ in $\alpha$, there must either be a $\hat{\alpha} \in\left(0, \underline{\alpha}_{g}\right)$ such that

$$
\int_{0}^{1} h(y) d y=\int_{0}^{1} h(y)\left[\frac{1}{2} d F^{\hat{\alpha}, h}(y)+\frac{1}{2} d F^{\hat{\alpha}, h}(y)\right]
$$

or, equivalently, using (5), such that

$$
\int_{0}^{1} h(y) d y=\int_{0}^{1} h(y) d F^{\hat{\alpha}, m}(y) .
$$


The latter equality implies that

$$
\underline{\alpha}_{h} \leq \hat{\alpha}<\underline{\alpha}_{g} .
$$

Or it must hold that

$$
\int_{0}^{1} h(y) d y \geq \int_{0}^{1} h(y)\left[\frac{1}{2} d F^{0, l}(y)+\frac{1}{2} d F^{0, h}(y)\right],
$$

which implies that NAM is an equilibrium without globalization, and thus $0=\underline{\alpha}_{h}<\underline{\alpha}_{g}$. In either case Inequality (A.1) holds.

Part (ii): Proof of Inequality (A.2)

We distinguish several cases with regard to the values of $\bar{\alpha}_{h}$ and $\bar{\alpha}_{g}$.

Case I: $\bar{\alpha}_{h}=0$

In this case, Inequality (A.2) holds trivially.

Case II: $\bar{\alpha}_{h}=1$

In this case, PAM is an equilibrium before globalization. Using Proposition 1, we obtain

$$
\int_{0}^{1} h(y) d y \geq \int_{0}^{1} h(y) d F^{\bar{\alpha}_{h}, m}(y)=\int_{0}^{1} h(y) d F^{1, m}(y) .
$$

Then (A.4) (see Lemma 1) together with Assumption 1 implies that

$$
\int_{0}^{1} g(h(y)) d y \geq \int_{0}^{1} g(h(y)) d F^{1, m}(y) .
$$

Hence, PAM is also an equilibrium after globalization, which means that $\bar{\alpha}_{g}=1$. That is, Inequality (A.2) holds.

Case III: $\bar{\alpha}_{h} \in(0,1)$

In this case, PAM is not an equilibrium before globalization, and there is an equilibrium with $\alpha>0$. Using Proposition 1, we obtain

$$
\int_{0}^{1} h(y) d y=\int_{0}^{1} h(y) d F^{\bar{\alpha}_{h}, m}(y) .
$$

Then (A.4) (see Lemma 1) together with Assumption 1 implies that

$$
\int_{0}^{1} g(h(y)) d y \geq \int_{0}^{1} g(h(y)) d F^{\bar{\alpha}_{h}, m}(y) .
$$

If (A.7) holds as equality, we are done since then $\bar{\alpha}_{h}$ is also an equilibrium after globalization, which means that Inequality (A.2) holds. Accordingly, consider that

$$
\int_{0}^{1} g(h(y)) d y>\int_{0}^{1} g(h(y)) d F^{\bar{\alpha}_{h}, m}(y) .
$$


Then, by continuity of $\int_{0}^{1} g(h(y)) d F^{\alpha, m}(y)$ in $\alpha$, there must either be $\hat{\alpha} \in\left(\bar{\alpha}_{h}, 1\right)$ such that

$$
\int_{0}^{1} g(h(y)) d y=\int_{0}^{1} g(h(y)) d F^{\hat{\alpha}, m}(y)
$$

which implies that

$$
\bar{\alpha}_{h}<\hat{\alpha} \leq \bar{\alpha}_{g}
$$

Or it must hold that

$$
\int_{0}^{1} g(h(y)) d y \geq \int_{0}^{1} g(h(y)) d F^{1, m}(y)
$$

which implies that PAM is an equilibrium with globalization, and thus $\bar{\alpha}_{g}=1>\bar{\alpha}_{h}$. In either case Inequality (A.2) holds.

Part (iii): Proof that Inequalities (A.1) and (A.2) can hold strictly

Finally, it remains to show that Inequalities (A.1) and (A.2) can hold strictly. To show this, we consider two examples. First, we find $h \in \mathcal{H}, g \in \mathcal{G}, F^{0, l}(\cdot)$ and $F^{0, h}(\cdot)$ such that

$$
\begin{aligned}
\int_{0}^{1} g(h(y)) \cdot \frac{1}{2}\left(d F^{0, l}(y)+d F^{0, h}(y)\right) & >\int_{0}^{1} g(h(y)) d y \\
& =\int_{0}^{1} h(y) d y \geq \int_{0}^{1} h(y) \cdot \frac{1}{2}\left(d F^{0,1}(y)+d F^{0, h}(y)\right) .
\end{aligned}
$$

Take $h(x)=x^{1 / 2}, g(x)=2(1-\delta) \cdot x^{4}+\delta \cdot x$, and

$$
f^{0, l}(y)= \begin{cases}\frac{1}{\epsilon} & \text { if } 0 \leq y \leq \epsilon \\ 0 & \text { otherwise }\end{cases}
$$

and

$$
f^{0, h}(y)=\left\{\begin{array}{ll}
\frac{1}{\epsilon} & \text { if } 1-\epsilon \leq y \leq 1 \\
0 & \text { otherwise }
\end{array} .\right.
$$

One can easily check that (A.9) holds if we take $\varepsilon>0$ and $\delta>0$ arbitrarily low. This means that $(\mathcal{W}, h, \overline{\mathcal{F}})$ satisfies $\mathrm{NAM}$, but $(\mathcal{W}, g \circ h, \overline{\mathcal{F}})$ does not. This means that for this economy, Inequality (A.1) holds strictly.

Second, we find $h \in \mathcal{H}, g \in \mathcal{G}$ and $F^{1, m}(\cdot)$ such that

$$
\int_{0}^{1} h(y) \cdot d F^{1, m}(y)>\int_{0}^{1} h(y) d y=\int_{0}^{1} g(h(y)) d y \geq \int_{0}^{1} g(h(y)) \cdot d F^{1, m}(y) .
$$

Let $h(x)=x^{1 / 2}$ and $g(x)=\frac{4(1-\delta)}{3} \cdot x^{2}+\delta \cdot x$, for $\delta>0$. Clearly, $h \in \mathcal{H}$ and $g \in \mathcal{G}$. It is a matter of simple algebra to verify that

$$
\int_{0}^{1} h(y) d y=\int_{0}^{1} g(h(y)) d y=\frac{2}{3} .
$$


Then, for a given $\varepsilon>0$, let

$$
d F^{1, m}(y)= \begin{cases}1 / \varepsilon & \text { if } 0.47-\varepsilon / 2 \leq y \leq 0.47+\varepsilon / 2 \\ 0 & \text { otherwise }\end{cases}
$$

If $\varepsilon>0$ and $\delta>0$ are arbitrarily low, we obtain

$$
\int_{0}^{1} g(h(y)) \cdot d F^{1, m}(y) \approx 0.63<\frac{2}{3}
$$

and

$$
\int_{0}^{1} h(y) \cdot d F^{1, m}(y) \approx 0.69>\frac{2}{3}
$$

Accordingly, $(\mathcal{W}, g \circ h, \overline{\mathcal{F}})$ satisfies PAM, but $(\mathcal{W}, h, \overline{\mathcal{F}})$ does not. This means that for this economy, Inequality (A.2) holds strictly. 


\section{B Additional Proofs (for online publication only)}

In this part of the appendix, we provide proofs of Propositions 1 and 2, which are based on Chade and Eeckhout (2020).

\section{B.1 Proof of Proposition 1}

In the following, we show the three parts of Proposition 1.

Proof of Part (i): In an equilibrium with positive assortative matching, $(\mu(1), \underline{w}, \bar{w})$, Conditions (10) reduce to Conditions (11) and (12). Because there is a continuum of workers - and hence every worker can always find another worker with whom to match-, it follows that

$$
\bar{w}=\frac{1}{2} \cdot \int_{0}^{1} h(y) d F^{1, h}(y)
$$

and

$$
\underline{w}=\frac{1}{2} \cdot \int_{0}^{1} h(y) d F^{1, l}(y)
$$

which yields Equations (14) and (15). With these expressions for wages, both Conditions (11) and (12) then reduce to the same condition, namely

$$
\frac{1}{2} \cdot V\left(t^{h} \mid \mu(1)\right)+\frac{1}{2} \cdot V\left(t^{l} \mid \mu(1)\right) \geq V\left(t^{m} \mid \mu(1)\right)
$$

Accordingly, there is an equilibrium with positive assortative matching if and only if (B.1) is satisfied. Now, note that for $\alpha=1$, Equation (5) reduces to

$$
1=\frac{1}{2} \cdot \frac{d F^{1, l}(y)}{d y}+\frac{1}{2} \cdot \frac{d F^{1, h}(y)}{d y} .
$$

Using the above equation and Equation (9), we can write

$$
\frac{1}{2} \cdot V\left(t^{h} \mid \mu(1)\right)+\frac{1}{2} \cdot V\left(t^{l} \mid \mu(1)\right)=\int_{0}^{1} h(y) \cdot\left(\frac{1}{2} \cdot d F^{1, l}(y)+\frac{1}{2} \cdot d F^{1, h}(y)\right)=\int_{0}^{1} h(y) d y
$$

and

$$
V\left(t^{m} \mid \mu(1)\right)=\int_{0}^{1} h(y) \cdot d F^{1, m}(y) \text {. }
$$

Finally, using the above two equations in (B.1), we obtain Condition (13). This concludes the proof of Part (i).

Proof of Part (ii): First of all, note that the same argument as in Part (i) implies that in an equilibrium $(\mu(\alpha), \underline{w}, \bar{w})$ with $\alpha \in(0,1)$, wages are given by (17) and (18). Further, with 
$\alpha \in(0,1)$, Conditions (10) reduce to

$$
\begin{aligned}
& V\left(t^{h} \mid \mu(\alpha)\right)-\bar{w}=V\left(t^{m} \mid \mu(\alpha)\right)-\underline{w}, \\
& V\left(t^{l} \mid \mu(\alpha)\right)-\underline{w}=V\left(t^{m} \mid \mu(\alpha)\right)-\bar{w} .
\end{aligned}
$$

Using the equilibrium wages given by Equations (17) and (18) as well as Equation (9), the above two equations can both be rearranged to

$$
\frac{1}{2} \cdot V\left(t^{h} \mid \mu(\alpha)\right)+\frac{1}{2} \cdot V\left(t^{l} \mid \mu(\alpha)\right)=V\left(t^{m} \mid \mu(\alpha)\right) .
$$

Finally, Equation (16) is easily obtained if we use Equation (9) again in combination with Equation (5). This finishes the proof of Part (ii).

Proof of Part (iii): With negative assortative matching, Conditions (10) reduce to

$$
\begin{aligned}
& V\left(t^{m} \mid \mu(0)\right)-\bar{w} \geq V\left(t^{l} \mid \mu(0)\right)-\underline{w}, \\
& V\left(t^{m} \mid \mu(0)\right)-\underline{w} \geq V\left(t^{h} \mid \mu(0)\right)-\bar{w} .
\end{aligned}
$$

On the one hand, if there is indeed an equilibrium $(\mu(0), \underline{w}, \bar{w})$, it must be that

$$
\underline{w}=V\left(t^{m} \mid \mu(0)\right)-\bar{w} .
$$

The reason is that wages of both members of a mixed team must add up to total expected payoffs of the team. Using Equation (9) on Equation (B.4), we obtain Equation (21), which is necessary for $(\mu(0), \underline{w}, \bar{w})$ to be an equilibrium. On the other hand, adding Conditions (B.2) and (B.3) yields the following necessary condition

$$
\frac{1}{2} \cdot V\left(t^{h} \mid \mu(0)\right)+\frac{1}{2} \cdot V\left(t^{l} \mid \mu(0)\right) \leq V\left(t^{m} \mid \mu(0)\right) .
$$

Condition (19) follows from using Equations (5) and (9) on (B.5). Finally, Condition (20) follows from noting that (i) if $\bar{w}<\frac{1}{2} V\left(t^{h} \mid \mu(0)\right)$, high-skilled workers would benefit from breaking apart from a mixed team and matching another high-skilled worker, (ii) if $\underline{w}<$ $\frac{1}{2} V\left(t^{l} \mid \mu(0)\right)$, low-skilled workers would benefit from breaking apart from a mixed team and then matching another low-skilled worker. Conversely, for any wages $\underline{w}$ and $\bar{w}$ that satisfy these two latter constraints plus Equation (B.4), no worker can profitably deviate from his current match, implying that the negative assortative matching $(\mu(0), \underline{w}, \bar{w})$ is indeed an equilibrium. ${ }^{46}$ This finishes the proof of Part (iii).

\footnotetext{
${ }^{46}$ Note that the set defined in (20) is non-empty by Equation (19).
} 


\section{B.2 Proof of Proposition 2}

Observe, on the one hand, that for no equilibrium with PAM to exist, we must have

$$
\begin{aligned}
\frac{1}{2} \cdot V\left(t^{l} \mid \mu(1)\right)+\frac{1}{2} \cdot V\left(t^{h} \mid \mu(1)\right) & =\int_{0}^{1} h(y) \cdot\left(\frac{1}{2} d F^{1, h}(y)+\frac{1}{2} d F^{1, l}(y)\right)=\int_{0}^{1} h(y) d y \\
& <\int_{0}^{1} h(y) d F^{1, m}(y)=V\left(t^{m} \mid \mu(1)\right),
\end{aligned}
$$

where the second equality follows from Equation (5). On the other hand, for no equilibrium with NAM to exist, we must have

$$
\begin{aligned}
V\left(t^{m} \mid \mu(0)\right) & =\int_{0}^{1} h(y) d F^{0, m}(y)=\int_{0}^{1} h(y) d y \\
& <\int_{0}^{1} h(y) \cdot\left(\frac{1}{2} d F^{0, h}(y)+\frac{1}{2} d F^{0, l}(y)\right)=\frac{1}{2} \cdot V\left(t^{l} \mid \mu(0)\right)+\frac{1}{2} \cdot V\left(t^{h} \mid \mu(0)\right) .
\end{aligned}
$$

Now, suppose that the above inequalities are both satisfied. Then, due to Assumption 4, there must exist $\alpha^{*} \in(0,1)$ such that

$$
V\left(t^{m} \mid \mu\left(\alpha^{*}\right)\right)=\frac{1}{2} \cdot V\left(t^{l} \mid \mu\left(\alpha^{*}\right)\right)+\frac{1}{2} \cdot V\left(t^{h} \mid \mu\left(\alpha^{*}\right)\right) .
$$

Using Equation (5) as well as the above equation, we obtain

$$
\begin{aligned}
\int_{0}^{1} h(y) d y & =\frac{\alpha^{*}}{2} \cdot V\left(t^{l} \mid \mu\left(\alpha^{*}\right)\right)+\frac{\alpha^{*}}{2} \cdot V\left(t^{h} \mid \mu\left(\alpha^{*}\right)\right)+\left(1-\alpha^{*}\right) \cdot V\left(t^{m} \mid \mu\left(\alpha^{*}\right)\right)=V\left(t^{m} \mid \mu\left(\alpha^{*}\right)\right) \\
& =\int_{0}^{1} h(y) d F^{\alpha^{*}, m}(y),
\end{aligned}
$$

and, hence, there is an equilibrium where workers are arranged according to $\mu\left(\alpha^{*}\right)$. This proves our claim that an equilibrium always exists under Assumption 4. 


\section{Further Derivations (for online publication only)}

In this appendix, we provide further details for some of the discussions in the main text.

\section{C.1 Uniqueness of equilibrium}

In this appendix, we discuss uniqueness of the equilibrium in our setup under Assumption 4. First, we show that NAM is the unique equilibrium if and only if

$$
V\left(t^{m} \mid \mu(\alpha)\right)>V\left(t^{m} \mid \mu(0)\right) \text { for all } \alpha \in(0,1] .
$$

Second, we show that PAM is the unique equilibrium if and only if

$$
V\left(t^{l} \mid \mu(\alpha)\right)+V\left(t^{h} \mid \mu(\alpha)\right)>V\left(t^{l} \mid \mu(1)\right)+V\left(t^{h} \mid \mu(1)\right) \text { for all } \alpha \in[0,1) .
$$

Third and last, we show that $\alpha^{*} \in(0,1)$ is the unique equilibrium if and only if

$$
\frac{1}{2} \cdot\left[V\left(t^{l} \mid \mu(\alpha)\right)+V\left(t^{h} \mid \mu(\alpha)\right)\right]\left\{\begin{array}{ll}
>V\left(t^{m} \mid \mu(\alpha)\right) & \text { if } \alpha \in\left[0, \alpha^{*}\right) \\
=V\left(t^{m} \mid \mu(\alpha)\right) & \text { if } \alpha=\alpha^{*} \\
<V\left(t^{m} \mid \mu(\alpha)\right) & \text { if } \alpha \in\left(\alpha^{*}, 1\right]
\end{array} .\right.
$$

Condition (C.1) requires that mixed teams expect the lowest payoff when all teams are mixed, while Condition (C.2) requires that the average payoff of a high- and a low-skilled team is lowest when no team is mixed. Condition (C.3) requires that - when viewed as a function of $\alpha$ - the expected value of a mixed team crosses the average expected value of high- and a low-skilled team exactly once and it does it from below.

In accordance with our previous discussion, we distinguish three cases. As the first case, we focus on NAM. To see that NAM is the unique equilibrium if and only if Condition (C.1) is satisfied, assume Condition (C.1). Then, for all $\alpha \in(0,1]$,

$$
\int_{0}^{1} h(y) d y=V\left(t^{m} \mid \mu(0)\right)<V\left(t^{m} \mid \mu(\alpha)\right)=\int_{0}^{1} h(y) d F^{\alpha, m}(y) .
$$

This means that there cannot be an equilibrium with $\alpha \in(0,1]$ - see Proposition 1-, and hence an equilibrium with NAM $(\alpha=0)$ is the only possibility. Conversely, suppose Condition (C.1) does not hold. Then, by Assumption 4, there either must be some $\alpha \in(0,1]$ such that

$$
\int_{0}^{1} h(y) d y=V\left(t^{m} \mid \mu(0)\right)=V\left(t^{m} \mid \mu(\alpha)\right)=\int_{0}^{1} h(y) d F^{\alpha, m}(y)
$$

and this $\alpha$ corresponds to an equilibrium - see Proposition 1 - or it must be the case that

$$
\int_{0}^{1} h(y) d y=V\left(t^{m} \mid \mu(0)\right)>V\left(t^{m} \mid \mu(1)\right)=\int_{0}^{1} h(y) d F^{1, m}(y),
$$


in which case PAM is an equilibrium. NAM is the only equilibrium in neither of the two cases.

As the second case, we focus on PAM. Then the fact that PAM is the unique equilibrium if and only if Condition (C.2) is satisfied can be proved using similar arguments as in the case of NAM just shown, if we use Equation (5).

As the third and last case, assume Condition (C.3), i.e., there is an interior equilibrium. Then, Proposition 1, Equation (5), and the definition of $V\left(t^{k} \mid \mu(\alpha)\right.$ (see Equation (9)) imply that $\alpha^{*}$ is the unique equilibrium. Conversely, suppose Condition (C.3) does not hold and $\alpha^{*}$ is an equilibrium. Then four cases need to be distinguished: First, if $V\left(t^{m} \mid \mu(\alpha)\right)$ and $\frac{1}{2} \cdot\left[V\left(t^{l} \mid \mu(\alpha)\right)+V\left(t^{h} \mid \mu(\alpha)\right)\right]$ never cross or touch, there cannot be an equilibrium with $\alpha \in(0,1)$, a contradiction. Second, if $V\left(t^{m} \mid \mu(\alpha)\right)$ and $\frac{1}{2} \cdot\left[V\left(t^{l} \mid \mu(\alpha)\right)+V\left(t^{h} \mid \mu(\alpha)\right)\right]$ touch at $\alpha^{*}$ but do not cross, then either PAM or NAM must also be an equilibrium. Third, if $V\left(t^{m} \mid \mu(\alpha)\right)$ crosses $\frac{1}{2} \cdot\left[V\left(t^{l} \mid \mu(\alpha)\right)+V\left(t^{h} \mid \mu(\alpha)\right)\right]$ exactly once, and it does it from above, then both PAM and NAM are also equilibria. Fourth and last, if $V\left(t^{m} \mid \mu(\alpha)\right)$ and

$\frac{1}{2} \cdot\left[V\left(t^{l} \mid \mu(\alpha)\right)+V\left(t^{h} \mid \mu(\alpha)\right)\right]$ cross more than once, then there is a $\hat{\alpha} \in[0,1], \hat{\alpha} \neq \alpha^{*}$, that is also an equilibrium. This completes the discussion about uniqueness of equilibria.

\section{C.2 Details on the discussions of Section 7.4 (core allocations)}

In this appendix we show that Corollary 1 also applies to core allocations. Starting from PAM, in our symmetric set-up it suffices to consider deviating coalitions that are composed of high- and low-skilled workers in equal shares. Let $S_{\alpha}$, with $\alpha \in[0,1]$, denote such a coalition with a measure $(1-\alpha)$ of both high- and low-skilled workers. A necessary condition for this coalition to be strictly better off by deviating from PAM and forming negatively assortatively matched teams is that

$$
(1-\alpha) \cdot \int_{0}^{1} h(y) d y<\int_{S_{\alpha}} V\left(t^{m} \mid \mu(\alpha)\right) d i=(1-\alpha) \cdot \int_{0}^{1} h(y) d F^{\alpha, m}(y),
$$

i.e. their joint expected payoff must be larger than their pro-rata share of the average payoff. The latter is the joint income if they stick with their current match under $\mu(1)$.

To analyze how our main results are affected when we consider deviations by any coalition instead of only deviations by a pair of individuals, we distinguish two cases: First, if coalition members anticipate the optimal response of (myopic) non-members to their rematching, Corollary 1 immediately applies. The reason is that in such a case, Condition (13) is 
necessary and sufficient for PAM to be a core allocation. ${ }^{47}$

Second, consider the case where coalition members can write a binding contract among themselves before the new matchings are formed and where they are myopic in that they do not anticipate any responses of non-members if they deviate themselves. ${ }^{48}$ In such case, PAM is a core allocation if and only if

$$
\int_{0}^{1} h(y) d y \geq \sup _{\alpha \in[0,1]} \int_{0}^{1} h(y) d F^{\alpha, m}(y)
$$

i.e. if and only if no coalition $S_{\alpha}, \alpha \in[0,1]$, is strictly better off forming mixed teams, given that everyone else sticks to their current match. Clearly, Condition (C.5) is stronger than Condition (13), so PAM will be an equilibrium whenever it is a core allocation but not necessarily vice versa. Yet, the effect of globalization as captured by function $g$ is the same: It allows PAM to be a core allocation under a wider set of parameters.

\section{C.3 Details on the discussions of Section 8.1.2 (patent race)}

In this appendix, we show that in case of a patent race as described in Section 8.1.2, the rank distribution of a mixed team satisfies Assumption 1 for any $\lambda^{l}, \lambda^{m}, \lambda^{h}>0$.

Note first that the rank of a team with skill level $k$ is distributed according to the cumulative distribution $F^{\alpha, k}(y)$, where for all $y \in[0,1]$,

$$
F^{\alpha, k}(y)=\operatorname{Pr}\left[r^{\alpha}(z) \leq y\right]=\operatorname{Pr}\left[z \geq r^{\alpha,-1}(y)\right]=1-B^{k}\left(r^{\alpha,-1}(y)\right)
$$

The second equality follows from $r^{\alpha \prime}(\cdot)<0$. Differentiating with respect to $y$ and applying

\footnotetext{
${ }^{47}$ To see this, suppose by way of contradiction that Condition (C.4) was satisfied for some $\alpha \in(0,1)$. Then it must be the case that a mixed team earns a higher expected payoff than the average of a high- and a low-skilled team. This means that individuals outside of coalition $S_{\alpha}$ would find it profitable to break apart from their current match under $\mu(1)$ and form negatively assorted teams as well. But this would increase the total share of teams that are matched negatively assortatively, inside and outside $S_{\alpha}$, to, say, $1-\alpha^{\prime}$. Let $\alpha^{*}$ be the largest $\alpha^{\prime} \in[0, \alpha]$ satisfying that

$$
(1-\alpha) \cdot \int_{0}^{1} h(y) d y=\int_{S_{\alpha}} V\left(t^{m} \mid \mu\left(\alpha^{\prime}\right)\right) d i=(1-\alpha) \cdot \int_{0}^{1} h(y) d F^{\alpha^{\prime}, m}(y) .
$$

Due to (5), $\alpha^{*}$ is well defined. Then, if the total share of teams that are matched negatively assortatively is $1-\alpha^{*}$, the coalition $S_{\alpha}$ that originally considered the possibility of deviating from PAM would no longer be strictly better off than when staying with their current matches given PAM. Similar arguments can be made for further iterations of deviations.

${ }^{48}$ Alternatively, we could assume that the timing of events is such that responses by non-members are not feasible.
} 
the chain rule, we obtain

$$
\begin{aligned}
f^{\alpha, k}(y) & =-b^{k}\left(r^{\alpha,-1}(y)\right) \cdot \frac{d}{d y} r^{\alpha,-1}(y) \\
& =-\lambda^{k} \exp \left(-\lambda^{k} r^{\alpha,-1}(y)\right) \cdot \frac{d}{d y} r^{\alpha,-1}(y) .
\end{aligned}
$$

Now, we want to show that the set

$$
\mathcal{A}:=\left\{y \in[0,1]: f^{\alpha, m}(y) \geq 1\right\}
$$

is compact and convex. To make progress, note first that this set is equal to

$$
\mathcal{A}=\{y \in[0,1]: \tilde{f}(y) \leq 1\}
$$

where

$$
\begin{aligned}
\tilde{f}(y) & :=\frac{(\alpha / 2) f^{\alpha, l}(y)+(\alpha / 2) f^{\alpha, h}(y)+(1-\alpha) f^{\alpha, m}(y)}{f^{\alpha, m}(y)} \\
& =\frac{(\alpha / 2) \lambda^{l} \exp \left(-\lambda^{l} r^{\alpha,-1}(y)\right)+(\alpha / 2) \lambda^{h} \exp \left(-\lambda^{h} r^{\alpha,-1}(y)\right)+(1-\alpha) \lambda^{m} \exp \left(-\lambda^{m} r^{\alpha,-1}(y)\right)}{\lambda^{m} \exp \left(-\lambda^{m} r^{\alpha,-1}(y)\right)} .
\end{aligned}
$$

The equivalence follows from the fact that the numerator in the first row is equal to one by Equation (5). The advantage of expressing the set $\mathcal{A}$ this way is that it allows us to get rid of the term $\frac{d}{d y} r^{\alpha,-1}(y)$ in Equation (C.6). Now, because $r^{\alpha,-1}(y)$ is strictly decreasing,

showing that the bounded set $\{y \in[0,1]: \tilde{f}(y) \leq 1\}$ is closed and convex is equivalent to proving that the set

$$
\{z \in[0, \infty): \tilde{f}(z) \leq 1\}
$$

is closed and convex, where $z=r^{\alpha,-1}(y)$. The desired result then follows from noting that

$$
\frac{d^{2}}{(d z)^{2}} \tilde{f}(z)=\frac{\alpha}{2}\left[\frac{\lambda^{l}}{\lambda^{m}}\left(\lambda^{m}-\lambda^{l}\right)^{2} \exp \left[\left(\lambda^{m}-\lambda^{l}\right) z\right]+\frac{\lambda^{h}}{\lambda^{m}}\left(\lambda^{m}-\lambda^{h}\right)^{2} \exp \left[\left(\lambda^{m}-\lambda^{h}\right) z\right]\right]>0 .
$$

This implies that $\tilde{f}(z)$ can cross one at most once from below.

\section{C.4 Details on the discussions of Section 8.2.2 (Melitz model)}

In this appendix, we show that in the variant of the Melitz (2003)-model considered in Section 8.2.2, conditional on the matching, the mass of entrants is independent of the trade environment.

The productivity of an entrepreneurial team $t^{k}$ with skill level $k \in\{l, m, h\}$ is drawn from a Pareto distribution with skill-dependent minimum-productivity level

$$
b^{k}(\varphi)= \begin{cases}\frac{\gamma \varphi^{k \gamma}}{\varphi^{\gamma+1}} & \text { if } \varphi \geq \underline{\varphi}^{k} \\ 0 & \text { otherwise }\end{cases}
$$


and where $\underline{\varphi}^{l} \leq \underline{\varphi}^{m} \leq \underline{\varphi}^{h}$. Ignoring knife-edge cases, free entry implies that the (expected) income of the lowest skilled entrepreneurs must equal the wage rate of workers, while higher skilled entrepreneurs earn positive rents. Note that this immediately implies that all high-skilled workers must work as entrepreneurs if some low-skilled workers are to work as entrepreneurs as well.

Now, to simplify exposition, suppose that entrepreneurial talent is scarce such that there are always some low-skilled teams. ${ }^{49}$ In equilibrium, free entry then implies that the ex-ante expected profit of a low-skilled team has to be equal to twice the wage rate for workers

$$
\sum_{j \in \mathcal{I}} \int_{\varphi_{i j}^{\star}}^{\infty}\left[\pi_{i j}(\varphi)-f_{i j} w_{i}\right] b^{l}(\varphi) d \varphi=2 w_{i}
$$

where $\mathcal{I}$ is the set of countries, $f_{i j}$ are the fixed cost in terms of domestic labor of serving destination country $j$ from country $i, w_{i}$ is the wage rate in country $i$, and $\pi_{i j}(\varphi)$ are the variable profits that a firm in country $i$ with productivity $\varphi$ can make when serving consumers in destination country $j . \varphi_{i j}^{\star}$ is the well-known productivity cutoff, i.e. a firm in country $i$ with productivity $\varphi_{i j}^{\star}$ just breaks even when serving destination country $j$. In equilibrium, a firm in country $i$ will serve destination $j$ if and only if it has productivity $\varphi \geq \varphi_{i j}^{\star}$. The labor market in country $i$ clears if

$$
M_{e i}\left[\sum_{j \in \mathcal{I}} \int_{\varphi_{i j}^{\star}}^{\infty}\left[l_{i j}^{v}(\varphi)+f_{i j}\right] b_{i}(\varphi) d \varphi\right]+M_{e i} f_{e i}=L_{i},
$$

where $M_{e i}$ is the total mass of entering firms, $l_{i j}^{v}(\varphi)$ the variable labor input for a firm in country $i$ with productivity $\varphi$ associated with serving destination country $j$, and $f_{e i}$ is the fixed cost of labor involved with founding a firm, i.e. $f_{e i}=2$ if entrepreneurial teams have two team members. $b_{i}(\varphi)$ is the productivity distribution of all firms in country $i$, which depends on the matching of entrepreneurs to teams. In particular, let $\alpha_{i}^{k}, k \in\{l, m, h\}$, be the share in country $i$ of entrepreneurial teams with skill level $k$. With this notation, we have that

$$
b_{i}(\varphi)=\alpha_{i}^{l} b^{l}(\varphi)+\alpha_{i}^{m} b^{m}(\varphi)+\alpha_{i}^{h} b^{h}(\varphi) .
$$

Combining Equations (C.7) to (C.10) and following steps as shown in the online appendix of Melitz and Redding (2014), we get $^{50}$

$$
M_{e i}=\frac{L_{i}}{f_{e i}}\left[1+\frac{\sigma(\gamma-1)+1}{\sigma-1} \cdot \frac{\alpha_{i}^{l} \underline{\varphi}^{l \gamma}+\alpha_{i}^{m} \underline{\varphi}^{m \gamma}+\alpha_{i}^{h} \underline{\varphi}^{h \gamma}}{\underline{\varphi}^{l \gamma}}\right]^{-1} .
$$

\footnotetext{
${ }^{49}$ That is, we consider the case where $M_{e_{i}}>\frac{L_{i}^{h}}{2}$, where $L_{i}^{h}$ denotes the mass of high-skilled labor in country $i$. This restriction is not essential for the following arguments and it can easily be dispensed with at the expense of additional notational complexity.

${ }^{50}$ For the case of $\underline{\varphi}^{l}=\underline{\varphi}^{m}=\underline{\varphi}^{h}$, Expression (C.11) reduces to Equation (22) in Melitz and Redding (2014).
} 
Hence, indeed, the mass of entering firms does not directly depend on the trade environment.

\section{C.5 Details on the discussions of Section 8.3.1 (technological change)}

Next, we show that the insight that technological change can increase the concentration of talent presented in Chade and Eeckhout (2020, Proposition 5) is a reflection of the much more general principle we identify. Chade and Eeckhout (2020, Section 5.1) consider workers that are of two types regarding skills. Then they analyze a two-stage game in which, in the first stage, these workers form teams of two. In the second stage, teams have the opportunity to invest in capital with cost of investment inversely proportional to $X^{\gamma}$, where $X$ is a measure of team skill. Payoffs are a function of the own investment and the investments of all other teams in the economy. They attribute the latter dependence to knowledge spillovers.

To analyze the role of technological change - an increase in $\gamma$ in their case - for equilibrium matching and inequality, Chade and Eeckhout (2020) limit attention to second stage equilibria such that the payoff of a team $k \in\{l, m, h\}$, given matching $\alpha$, can be written as

$$
V\left(X^{k} \mid \alpha\right)= \begin{cases}A^{2} \cdot\left(X^{l}\right)^{\gamma} \cdot(\lambda+1-\alpha / 2)^{2} / 2 & \text { if } k=l \\ A^{2} \cdot\left(X^{m}\right)^{\gamma} \cdot(\lambda+\alpha / 2)^{2} / 2 & \text { if } k=m, \\ A^{2} \cdot\left(X^{h}\right)^{\gamma} \cdot \lambda^{2} / 2 & \text { if } k=h\end{cases}
$$

for some constants $\lambda>0$ and $X^{h}>X^{m}>X^{l}>0$, and where $2 X^{m}=X^{h}+X^{l}$. From (C.12), we can immediately see that there are two potentially opposing forces with respect to the expected payoff for a mixed team relative to the average expected payoff of a high-skilled team and a low-skilled team. On the one hand, for $\gamma>1$ it holds that

$$
\left(X^{l}\right)^{\gamma}+\left(X^{h}\right)^{\gamma}>2 \cdot\left(X^{m}\right)^{\gamma},
$$

which is a force in favor of positive assortative matching. On the other hand, for $\alpha$ sufficiently close to 1

$$
(\lambda+1-\alpha / 2)^{2} / 2+\lambda^{2} / 2<(\lambda+\alpha / 2)^{2},
$$

which is a force against positive assortative matching. This opposing force-which is due to knowledge spillovers - is stronger the larger $\alpha$. We stress that the latter parameter denotes the share of positively assortatively matched teams.

To derive their results, Chade and Eeckhout (2020) further restrict the parameter space such that there is a unique stable matching in the first stage with $\alpha \in(0,1)$. That is, it must be the case that there is $\alpha^{*} \in(0,1)$ such that

$$
H\left(\alpha^{*}, \gamma\right):=V\left(X^{l} \mid \alpha^{*}\right)+V\left(X^{h} \mid \alpha^{*}\right)-2 \cdot V\left(X^{m} \mid \alpha^{*}\right)=0 .
$$


Note that

$$
d H(\alpha, \gamma)=\frac{\partial H(\alpha, \gamma)}{\partial \alpha} \cdot d \alpha+\frac{\partial H(\alpha, \gamma)}{\partial \gamma} \cdot d \gamma
$$

For a given $\alpha^{*}$, their parametric restrictions imply that $\frac{\partial H(\alpha, \gamma)}{\partial \alpha} \leq 0$ and $\frac{\partial H(\alpha, \gamma)}{\partial \gamma} \geq 0$. Then $d H\left(\alpha^{*}, \gamma\right)=0$ implies that $\alpha^{*}$ must increase if $\gamma$ increases, i.e. the equilibrium share of positively assortatively matched teams increases in response to a higher $\gamma$.

To see the connection to our work, note that for $\alpha^{*}$ given, (C.12) describes a discrete function that maps team skills $X^{k}(k \in\{l, m, h\})$ into payoffs. This function is convex if and only if

$$
H\left(\alpha^{*}, \gamma\right) \geq 0
$$

and it is concave otherwise. Then the fact that $\frac{\partial H(\alpha, \gamma)}{\partial \gamma} \geq 0$ implies that $\gamma$ increases the convexity of payoffs, taken the aggregate matching as fixed (i.e. fixing $\alpha$ ). Moreover, since team skills are deterministic, for any given $\alpha$ we can view teams' skills as realizations of Dirac deltas (at $X^{l}, X^{m}$, and $X^{h}$, for teams of type $t^{l}, t^{m}$, and $t^{h}$, respectively) that satisfy a single-crossing condition analogous to Assumption $1^{\prime \prime \prime}$ - see Section 7.3. Hence, we could apply Lemma 1 to conclude that if, given $\alpha^{*}$, the mixed team is no better off than positively assortatively matched teams before the change in $\gamma$ (as implied by Equation (C.13)), it cannot be better off after the change in $\gamma$. This would eventually lead to the conclusion that the concentration of talent must increase - see Theorem 1. Of course, in this case the application of Lemma 1 would not be necessary as the implication directly follows from their assumptions on $H(\cdot)$. This means that the contribution in Chade and Eeckhout (2020) is rather to find the parametric restriction that guarantees the convex transformation of payoffs as captured by $H(\cdot)$ (see Equation (C.13)). The key point to note here is therefore not the applicability of Lemma 1, but that the comparative statics on matching outcomes in Chade and Eeckhout (2020, Proposition 5) is a reflection of the much more general principle we identify and that applies in a broad variety of contexts over and above the specific example they consider.

\section{C.6 Details on the discussions of Section 8.3.4 (taxation)}

Next, we show that a convex transform of the tax schedule as discussed in Section 8.3.4 has two opposing effects on the likelihood that PAM is an equilibrium. To this end, we consider a change from a flat-rate tax to a convex tax schedule. Taxes are applied at the individual level, so the net income of a worker who receives payout $x$ is given by

$$
x-T(x)
$$

where $x$ depends on the competition outcomes and $T(x)$ is a tax schedule. How does the schedule change the conditions for PAM? 
To answer the above question, suppose that workers are arranged according to $\mu(1)$ (i.e, PAM), and that team members share the joint payoff according to a fixed rule. Then, the expected (net) payoff of a high-skilled worker is

$$
\int_{0}^{1}\left[\frac{h(y)}{2}-T\left(\frac{h(y)}{2}\right)\right] d F^{1, h}(y)
$$

while the expected (net) payoff of a low-skilled worker is

$$
\int_{0}^{1}\left[\frac{h(y)}{2}-T\left(\frac{h(y)}{2}\right)\right] d F^{1, l}(y) .
$$

Then, consider that a high-skilled worker $i$ wants to form a team $t^{m}$ with a low-skilled worker $j$. Clearly, $i$ will offer $j$ a share $1-\omega$, with $\omega \in[0,1]$, that yields $j$ his/her current expected payoff, i.e.,

$$
\int_{0}^{1}[(1-\omega) h(y)-T((1-\omega) h(y))] d F^{1, m}(y)=\int_{0}^{1}\left[\frac{h(y)}{2}-T\left(\frac{h(y)}{2}\right)\right] d F^{1, l}(y) .
$$

This means that $i$ expects a payoff equal to

$$
\int_{0}^{1}[\omega h(y)-T(\omega h(y))] d F^{1, m}(y)
$$

If $\mu(1)$ is stable, it must therefore be the case that

$$
\int_{0}^{1}[\omega h(y)-T(\omega h(y))] d F^{1, m}(y) \leq \int_{0}^{1}\left[\frac{h(y)}{2}-T\left(\frac{h(y)}{2}\right)\right] d F^{1, h}(y) .
$$

Using Condition (C.15) and the fact that

$$
\frac{1}{2} \cdot f^{1, l}(y)+\frac{1}{2} \cdot f^{1, h}(y)=1
$$

we obtain

$$
\begin{aligned}
& \int_{0}^{1}\left[\frac{h(y)}{2}-T\left(\frac{h(y)}{2}\right)\right] d F^{1, h}(y) \\
= & \int_{0}^{1} h(y) d y-\int_{0}^{1} 2 T\left(\frac{h(y)}{2}\right) d y-\int_{0}^{1}[(1-\omega) h(y)-T((1-\omega) h(y))] d F^{1, m}(y) .
\end{aligned}
$$

Condition (C.16) then reduces to

$$
\begin{aligned}
& \int_{0}^{1} h(y) d y-\int_{0}^{1} h(y) d F^{1, m}(y) \\
\geq & \int_{0}^{1} 2 T\left(\frac{h(y)}{2}\right) d y-\int_{0}^{1}(T(\omega h(y))+T((1-\omega) h(y))) d F^{1, m}(y) .
\end{aligned}
$$


We stress that $\omega$ depends on $T(\cdot)$, as shown by Equation (C.15). Note that in the case of a flat-rate tax schedule, i.e., if $T(x)=\tau x$, Equation (C.17) reduces to the familiar condition

$$
\int_{0}^{1} h(y) d y-\int_{0}^{1} h(y) d F^{1, m}(y) \geq 0 .
$$

This means that flat rate taxes do not affect the incentives of workers to match according to PAM. However, this is no longer true if $T(x)$ is convex, and two opposing effects can be distinguished. On the one hand, mixed teams are more likely to achieve mid-range ranks, which ceteris paribus implies that they are less affected by the convex tax scheme and, hence, makes PAM less likely. On the other hand, mixed teams split the team payoff unequally $\left(\omega \geq \frac{1}{2}\right)$, which implies that for any given rank $y \in[0,1]$ a mixed team pays higher taxes than positively assortatively matched teams

$$
2 T\left(\frac{h(y)}{2}\right) \leq T(\omega h(y))+T((1-\omega) h(y)) .
$$

Ceteris paribus this implies that a change to a convex tax schedule increases the incentives for workers to match positively assortatively. Which of the two effects dominates depends on the exact circumstances under consideration. 